\title{
X-ray and optical observations of 1RXS J154814.5-452845: A new intermediate polar with soft $X$-ray emission ${ }^{\star}$
}

\author{
F. Haberl ${ }^{1}$, C. Motch $^{2}$, and F.-J. Zickgraf ${ }^{3}$ \\ 1 Max-Planck-Institut für extraterrestrische Physik, Giessenbachstraße, 85748 Garching, Germany \\ 2 Observatoire de Strasbourg, 11, rue de l'Universite, 67000 Strasbourg, France \\ 3 Hamburger Sternwarte, Gojenbergsweg 112, 21029 Hamburg, Germany
}

Received 21 December 2001/ Accepted 5 March 2002

\begin{abstract}
We report the identification of the ROSAT all-sky survey source 1RXS J154814.5-452845 as new intermediate polar and present the results from follow-up optical and X-ray observations. The source shows pulsations with a period of $693 \mathrm{~s}$ both in the optical and X-ray light curves and the detection of a synodic frequency strongly suggests that this is the rotation period of the white dwarf. Although the one day aliasing and the sparse optical data coverage does not allow to unambiguously identify the orbital period, the most likely values of $9.37 \mathrm{~h}$ and $6.72 \mathrm{~h}$ add 1RXS J154814.5-452845 to the intermediate polars with the longest orbital periods known. The optical spectrum displays features from the late type secondary and shows the presence of broad absorption lines at $\mathrm{H} \beta$ and higher order Balmer lines which may be a signature of the white dwarf atmosphere, very similar to V 709 Cas (RX J0028.8+5917 Bonnet-Bidaud et al. 2001). The average X-ray spectra as obtained by the EPIC instruments on board XMM-Newton show hard emission typical for this class of objects but also the presence of soft blackbody-like emission similar to that seen from soft intermediate polars and thought to arise from the white dwarf surface heated by the hard X-rays. The best fit model comprises thermal emission from multi-temperature plasma in collisional ionization equilibrium with a continuous temperature distribution up to a maximum of $\sim 60 \mathrm{keV}$, an Fe fluorescence line at $6.4 \mathrm{keV}$ and with equivalent width of $260 \mathrm{eV}$ and a blackbody component with $\mathrm{kT}$ of $86 \mathrm{eV}$. The hard X-ray emission is absorbed by matter covering $47 \%$ of the X-ray source with an equivalent hydrogen density of $\sim 10^{23} \mathrm{~cm}^{-2}$. The remaining hard emission is absorbed by a much reduced column density of $1.5 \times 10^{21} \mathrm{~cm}^{-2}$ as is the soft blackbody emission. Pulse-phase spectroscopy around spin maximum and minimum reveals that the flux variations are mainly caused by a change in the temperature distribution with higher intensity (a factor of $\sim 3$ in the $1 \mathrm{keV}$ emission) seen from the lower temperature plasma during spin maximum. The absorption in the high column density matter only decreases marginally during spin maximum. The emission characteristics are consistent with the accretion curtain scenario and features in the X-ray pulse profiles indicate that we observe one pole of the white dwarf and our line of sight is nearly parallel to the curtain at spin minimum while at maximum we have a more direct view to the cooling post shock accretion flow.
\end{abstract}

Key words. binaries: close - stars: individual: 1RXS J154814.5-452845 - stars: novae, cataclysmic variables $\mathrm{X}$-rays: stars

\section{Introduction}

The ROSAT Galactic Plane Survey project (RGPS, Motch et al. 1991) to identify X-ray sources detected at low galactic latitudes $\left(|b|<20^{\circ}\right)$ led to the discovery of new cataclysmic variables (Haberl \& Motch 1995; Motch et al. 1996). Cataclysmic variables (CVs) are close binary systems in which the low mass secondary star fills its Roche

Send offprint requests to: F. Haberl,

e-mail: fwh@mpe.mpg.de

* Based on observations with XMM-Newton, an ESA Science Mission with instruments and contributions directly funded by ESA Member states and the USA (NASA) and on observations collected at the European Southern Observatory. lobe and transfers matter onto the white dwarf primary. Depending on the magnetic field strength of the white dwarf the accretion flows via an accretion disc or directly couples onto the magnetic field lines of the white dwarf in the case of a strong magnetic field. Magnetic cataclysmic variables are usually divided into polars (AM Her systems) in which the strong magnetic field forces the two stars into co-rotation and intermediate polars with shorter white dwarf spin periods of typically a few hundred seconds (IPs, for reviews of this latter sub-class see Patterson 1994; Hellier 1995, 1999). IPs accrete from the accretion disc via an azimuthally extended accretion curtain which causes high photo-electric absorption. 
Table 1. ROSAT hardness ratios of IPs.

\begin{tabular}{lcc}
\hline \hline IP & $H R 1$ & $H R 2$ \\
\hline 1RXS J154814.5-452845 & $+0.80 \pm 0.07$ & $+0.06 \pm 0.11$ \\
RX J0028.8+5917 & $+0.95 \pm 0.01$ & $+0.37 \pm 0.01$ \\
RX J1712.6-2414 & $+0.92 \pm 0.01$ & $+0.32 \pm 0.01$ \\
RE 0751+14 & $-0.83 /-0.79$ & $-0.09 /-0.15$ \\
RX J0558.0+5353 & $-0.45 \pm 0.01$ & $-0.09 \pm 0.02$ \\
\hline
\end{tabular}

The X-ray spectra of most intermediate polars show a hard, absorbed bremsstrahlung continuum plus emission lines from highly ionized iron. This emission originates from the matter which free-falls towards the white dwarf creating a strong shock near the surface with temperatures kT up to several tens of keV. The hard X-rays heat the white dwarf surface, creating a soft blackbody-like component as is generally observed in polars (Beuermann \& Thomas 1993) where the accretion stream is of pencillike geometry. Additional heating may be caused by dense blobs in the accretion flow which directly penetrate the white dwarf atmosphere. The temperature of the emission is thought to decrease with increasing size of the accretion region on the white dwarf (King \& Lasota 1990). Therefore, the absence of detectable soft X-ray emission in classical IPs may be explained by a too low temperature and/or suppression of soft X-rays due to considerable absorption. The discovery of little absorbed, soft X-ray emission from a small number of "soft" intermediate polars in ROSAT data (Mason et al. 1992; Haberl et al. 1994) together with relatively high magnetic field strengths (e.g. Potter et al. 1997) which collimates the accretion stream is consistent with this picture. Here we present the discovery of a new cataclysmic variable. Optical observations together with detailed X-ray follow-up using ROSAT and XMM-Newton clearly identify 1RXS J154814.5-452845 as new intermediate polar.

\section{Discovery in the ROSAT all-sky survey}

ROSAT detected 1RXS J154814.5-452845 during the allsky survey (Trümper 1982) with $0.54 \pm 0.06 \mathrm{cts} \mathrm{s}^{-1}$ in the PSPC detector (Pfeffermann et al. 1987). The source is listed in the bright source catalogue (Voges et al. 1999) with hardness ratios (defined as $H R 1=(H-S) /(S+H)$ and $H R 2=(H 2-H 1) /(H 1+H 2)$ where $S, H, H 1$ and $H 2$ denote count rates in the $0.1-0.4 \mathrm{keV}, 0.5^{-}$ $2.0 \mathrm{keV}, 0.5-0.9 \mathrm{keV}$ and $0.9-2.0 \mathrm{keV}$ bands, respectively) which indicate a moderately hard X-ray spectrum in the ROSAT band. Table 1 compares the hardness ratios of 1RXS J154814.5-452845 to four other ROSAT-discovered IPs extracted from the RRA PSPC source catalogue from pointed ROSAT observations (ROSAT Consortium 2000). Classical IPs like RX J0028.8+5917 and RX J1712.6-2414 show high absorption, for which HR1 is very sensitive to with values approaching +1 . The "soft" IPs RE $0751+14$

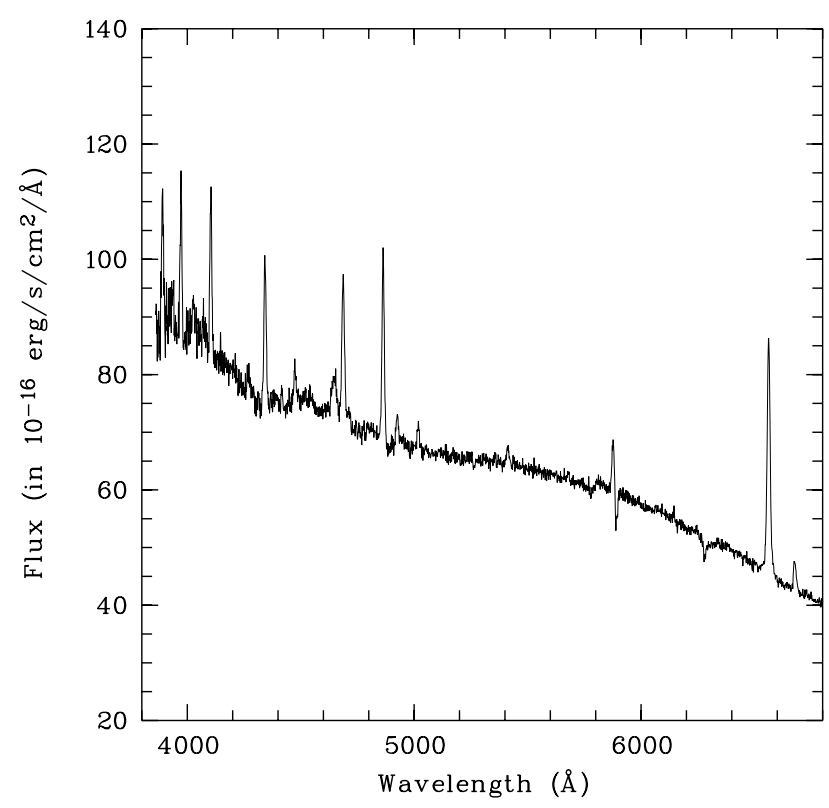

Fig. 1. Mean optical spectrum of the optical counterpart of 1RXS J154814.5-452845 as observed in June 1997.

and RX J0558.0+5353 show little absorption (negative $H R 1$ ) but also softer intrinsic spectra (negative $H R 2$ ) caused by a strong soft blackbody spectral component which dominates the spectrum in the ROSAT band. The hardness ratios of 1RXS J154814.5-452845 are between those of the two groups, suggesting less absorption than seen from classical IPs and/or the existence of a soft spectral component.

\section{Optical observations}

\subsection{Optical identification}

The source was included in the RGPS bright source sample for optical identification. We observed the field of 1RXS J154814.5-452845 for the first time on 1997, June 6 with DFOSC and the LORAL $2 \mathrm{k} \times 2 \mathrm{k}$ CCD at the ESODanish $1.54 \mathrm{~m}$ telescope. The X-ray source was readily identified with a $V \sim 14.6$ blue object exhibiting Balmer, He I, HeII and NIII-CIII $\lambda \lambda 4640-60$ lines in emission. We show in Fig. 1 the mean of two $10 \mathrm{mn}$ long spectra obtained with a $2.5^{\prime \prime}$ slit through grism \#7 $(\lambda \lambda 3860-6810 \AA$; $10 \AA$ FWHM resolution). Figure 2 shows the position of the optical counterpart on a $V$ band image.

\subsection{Follow-up optical observations}

We organized an optical observation campaign in May 1998 involving both photometric and spectroscopic monitoring at the ESO-Dutch $0.9 \mathrm{~m}$ and ESO-Danish $1.54 \mathrm{~m}$ telescopes, respectively. During three consecutive nights from May 7 to 9 UT we obtained optical $V$ band photometry using the standard CCD camera equipped with the TK512CB \# 33 CCD. In order to ensure the best compromise between time resolution and observing efficiency the CCD was windowed and the exposure time was 


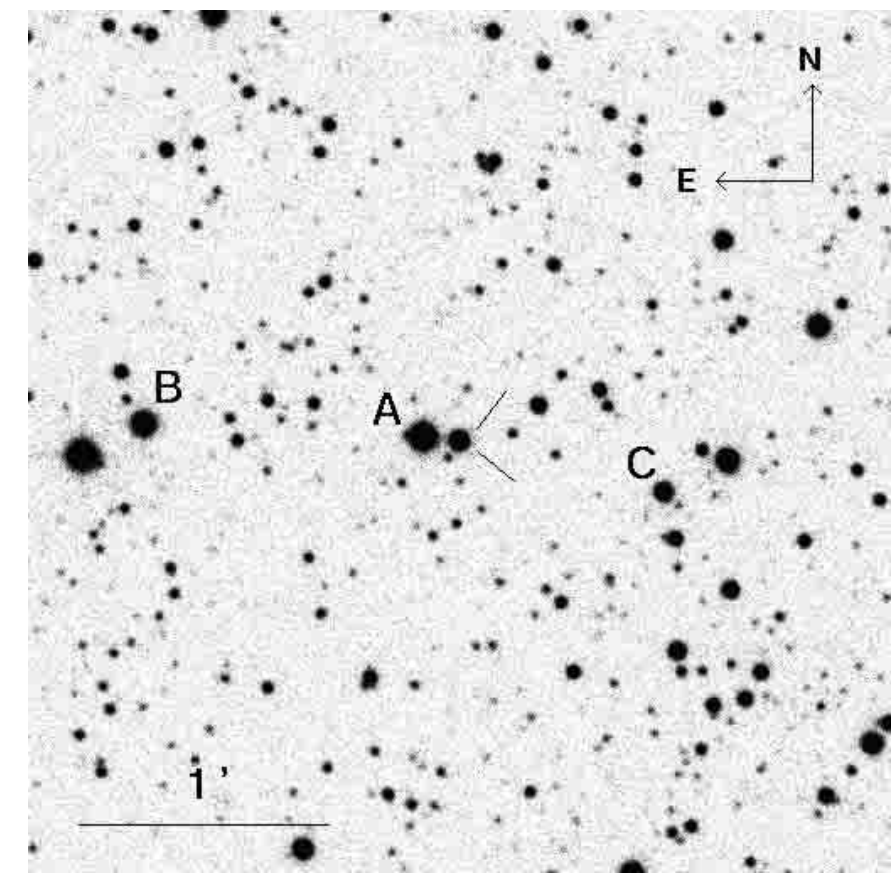

Fig. 2. $V$ band finding chart for 1RXS J154814.5-452845. The positions of the three comparison stars, A, B and C used for differential photometry are also shown.

set to $30 \mathrm{~s}$. With this instrumental setting, the mean time between two consecutive exposures was $\sim 53 \mathrm{~s}$. A total of 753 frames were acquired over $11.5 \mathrm{~h}$. Raw images were corrected for bias and flat-field using standard MIDAS procedures. Instrumental magnitudes were computed using the SExtractor package (Bertin \& Arnouts 1996) for the optical counterpart and 3 comparison stars, named A, $\mathrm{B}$ and $\mathrm{C}$ whose positions are indicated in Fig. 2. Weather conditions were not photometric during the first and the third night. However, the accuracy of the differential photometry remains good with average errors below the $1 \%$ level. From standard star measurements in June 1997 and May 1998 we derive the following magnitudes for the comparison stars; $V_{\mathrm{A}}=12.94 \pm 0.04, V_{\mathrm{B}}=13.51 \pm 0.04$ and $V_{\mathrm{C}}=15.00 \pm 0.04$. The photometric uncertainty is dominated by the colour transformation. The mean magnitude of 1RXS J154814.5-452845 recorded in May 1998 was $V=14.61$, somewhat fainter than in June 1997, $V=14.46$. Spectroscopic observations took place from May 15 till 17 UT. At the ESO-Danish $1.54 \mathrm{~m}$ telescope, we used the same DFOSC setting as in 1997 but with a smaller entrance slit of $1.5^{\prime \prime}$. With grism \#7 the wavelength range was $\lambda \lambda 3860-6840 \AA$ with a $5.7 \AA$ FWHM resolution). Because of the smaller slit used in 1998 than in 1997, the spectral resolution improved but no accurate flux calibration could be established. In particular, differential refraction distorted the shape of the flux calibrated spectrum. The first spectrum had an exposure time of $20 \mathrm{mn}$ and the remaining 8 were $15 \mathrm{mn}$ long. Arcs from $\mathrm{He}$ and Ne lamps provided the wavelength scale. As for photometric data, MIDAS procedures were used to cor- rect 2 -d frames and extract and calibrate spectra in wavelength and flux.

\subsection{Spectroscopic analysis}

\subsubsection{The orbital period}

Radial velocities of Balmer and of He II $\lambda 4686$ emission lines vary relatively slowly within a single night. The total amplitude is $200 \mathrm{~km} \mathrm{~s}^{-1}$ for $\mathrm{H} \alpha$. Balmer and He II velocities seem roughly in phase although there are large differences in mean velocities, higher Balmer lines being more blue shifted than $\mathrm{H} \alpha$. We list in Table 2 the measured emission line velocities. In order to search for orbital motion we computed periodograms for the various lines using the method outlined in Lomb (1976) and Scargle (1982) which is suitable for unequally spaced data. Spectroscopic data are consistent with a rather long orbital period, larger than $5 \mathrm{~h}$. Because of the strong one day aliasing and sparse coverage it is not possible to unambiguously derive the orbital period. We find that the two most probable periods are $P=9.37 \pm 0.69 \mathrm{~h}$ and $P=6.72 \pm 0.32 \mathrm{~h}$. We show in Fig. 3 the $\mathrm{H} \alpha$ radial velocity curve folded with a period of $9.65 \pm 0.03 \mathrm{~h}$ which gives the best sine fit. At this period, He II $\lambda 4686$ velocities have a more structured pattern of variation. However, the overall shape of the radial velocity curve changes with period and clearly more spectroscopic data are needed to determine the true orbital period and velocity structure.

A rather strong indication in favour of a long orbital period is the presence of several metallic absorption lines in the red part of the spectrum. We compare in Fig. 4 the red part of the mean optical spectrum obtained in 1998 with a template K4 III spectrum extracted from the Jacoby et al. (1984) library of stellar spectra. The resolution of the library spectra $(\sim 4.5 \AA)$ compares well with that of our observations. In order to better highlight the similarities we diluted the late type spectrum by a factor 5 using a constant continuum and plotted together the rectified spectra normalized to the average continuum. The Mg " $b$ " bands and the $\lambda 5206,5270,6362$, $6497 \AA$ atomic line blends are visible as well as the Ca I $\lambda 6162 \AA$ line. The Na I doublet is well marked, however, part of its strength could be due to interstellar absorption as indicated by the presence of a relatively deep $\lambda 5780 \AA$ band. The large absorption at $\lambda 6280 \AA$ is probably a mixture of telluric $\mathrm{O} 2$ and diffuse interstellar bands. It is not possible to classify the late type spectrum with great accuracy. Torres-Dodgen \& Weaver (1993) have identified useful lines for classifying stars in the near-infrared. The absence of $\mathrm{TiO}$ bands at $\lambda 6080-6390 \AA$ indicates a spectrum earlier than K7V. The marked $\lambda 6497 \AA$ blend and Ca I line suggests an early $\mathrm{K}$ type. Finally, the presence of the Fe I, TiI Cr I blend at $\lambda 6362 \AA$ may indicate an evolved type. Unfortunately, the absorption line depths on individual $15 \mathrm{mn}$ long spectra are not strong enough to measure a reliable radial velocity curve of the late type companion. 
Table 2. Radial velocities corrected at barycentre of solar system in $\mathrm{km} \mathrm{s}^{-1}$.

\begin{tabular}{rrrrr}
\hline \hline $\begin{array}{r}\text { Julian date } \\
-2400000.5 \mathrm{~d}\end{array}$ & $\mathrm{H} \alpha$ & $\mathrm{H} \beta$ & $\mathrm{H} \gamma$ & $\begin{array}{r}\mathrm{He} \mathrm{II} \\
\lambda 4686\end{array}$ \\
\hline 50948.28693 & +94.1 & +33.8 & +12.0 & +28.9 \\
50948.32557 & +46.6 & -30.2 & -34.1 & -9.8 \\
50948.36179 & +2.9 & -61.5 & -69.5 & -23.9 \\
50948.41196 & -62.4 & -102.8 & -121.1 & -59.5 \\
50949.23678 & -99.6 & -141.2 & -167.1 & -119.4 \\
50949.29622 & -91.7 & -120.1 & -132.0 & -101.5 \\
50949.34037 & -53.3 & -45.7 & -52.4 & -89.3 \\
50949.40147 & +41.7 & +43.8 & +11.3 & +17.1 \\
50950.41219 & -56.5 & -69.7 & -60.2 & -28.9 \\
\hline
\end{tabular}

A spectral type hotter than $\mathrm{K} 7$ and possibly early $\mathrm{K}$ is compatible with the long orbital period derived from our spectroscopy. The mean density of the Roche lobe filling star can be expressed as $\rho \approx 115 \mathrm{P}_{\mathrm{hr}}^{-2} \mathrm{~g} \mathrm{~cm}^{-3}$ (Frank et al. 1992). The shortest possible period of $6.72 \mathrm{~h}$ implies densities comparable to those of a main sequence K5 star. If the orbital period is $9.37 \mathrm{~h}$ a main sequence star would have a $\mathrm{G}$ type. Although this cannot be ruled out completely from our data, the requirement for stable mass transfer of $q=M_{2} / M_{1} \leq 5 / 6$ (Frank et al. 1992) would rather suggest a slightly evolved $\mathrm{K}$ secondary instead. This picture is consistent with the spectral type distribution of secondary stars in cataclysmic variables compiled by Smith \& Dhillon (1998) which shows that no system below $4.3 \mathrm{~h}$ period has a secondary earlier than K.

Standard evolutionary sequences for unevolved donor stars undergoing mass-transfer predict a $\sim \mathrm{K} 2$ spectral type if the orbital period of the $\mathrm{CV}$ is $6.72 \mathrm{~h}$ (Baraffe \& Kolb 2000). Alternatively, a $\mathrm{K}$ type secondary in a $9.37 \mathrm{~h}$ period CV would only be possible if the mass donor star is slightly evolved (Beuermann et al. 1998). Therefore, in the absence of confirmed orbital period and secondary mass estimates, the evolutionary status of the mass donor star cannot be determined.

\subsubsection{Spectral signature of the white dwarf?}

Another outstanding feature of 1RXS J154814.5-452845 is the presence of broad absorption lines at $\mathrm{H} \beta$ and higher order Balmer lines. Troughs are seen up to $\mathrm{H} \epsilon$ in the blue part of the spectrum (see Fig. 5). In that respect, the spectrum of 1RXS J154814.5-452845 is strikingly similar to that of the ROSAT discovered classical intermediate polar V 709 Cas (RX J0028.8+5917 Bonnet-Bidaud et al. 2001). These authors argue that the broad absorptions are the signature of the non heated part of the accreting star and derive $\log g=8$ and $T=23000 \mathrm{~K}$ for the DA white dwarf. Similar conclusions may be driven for 1RXS J154814.5-452845. One should however take this interpretation with some caution since similar broad Balmer lines in absorption with superposed narrow emissions are

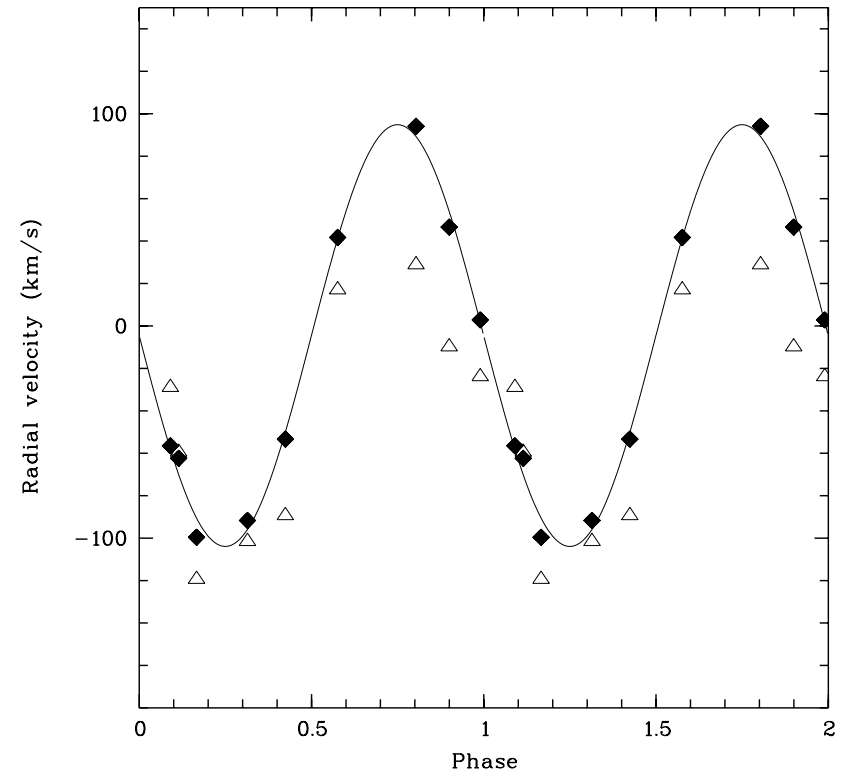

Fig. 3. Example of radial velocity curves: $\mathrm{H} \alpha$ velocities (filled diamond) folded with the best fit sine of period $P=9.65 \pm$ $0.03 \mathrm{~h}$. Other periods close to $6.7 \mathrm{~h}$ are also consistent with the spectroscopic data. He II $\lambda 4686$ radial velocities shown as open triangles follow a less regular pattern of variation.

sometimes seen from accretion discs in UX UMa stars (see e.g. and references therein Warner 1995).

\subsection{Photometric timing analysis}

We show in Fig. 6 the $V$ light curve of 1RXS J154814.5-452845 for the three consecutive nights of observations. Significant photometric activity occurs all along the light curve on time scales of minutes. No conspicuous large long term variations indicating a possible orbital modulation is seen although the hollow at the beginning of the second night may represent some minimum. In order to search for coherent modulations we computed the Lomb-Scargle periodogram of the entire time series. Figure 7 shows the resulting power spectrum. A clear signal is detected at $f=124.72 \pm 0.10$ cycle/day or $P=692.75 \pm 0.56 \mathrm{~s}$. This period is consistent with the most probable X-ray period derived from the long HRI observation $(P=693.3 \pm 1.1 \mathrm{~s}$, see below $)$. Although the two closest one day aliases of the photometric period cannot strictly be ruled out on a statistical basis, none of them appears consistent with any of the possible HRI aliases (see Fig. 8). The natural aliasing frequencies of ground based and space borne instruments are obviously different. It is therefore very likely that the optical pulsation occurs at the same frequency as in X-ray and probably represents the spin period of the accreting white dwarf unless the accretion is disc-less as supposed in RX J1712.6-2414 for instance (Buckley et al. 1997). The absence of large power at harmonic frequencies is consistent with the sinusoidal shape of the folded light curve shown in Fig. 9. The full amplitude of the folded 


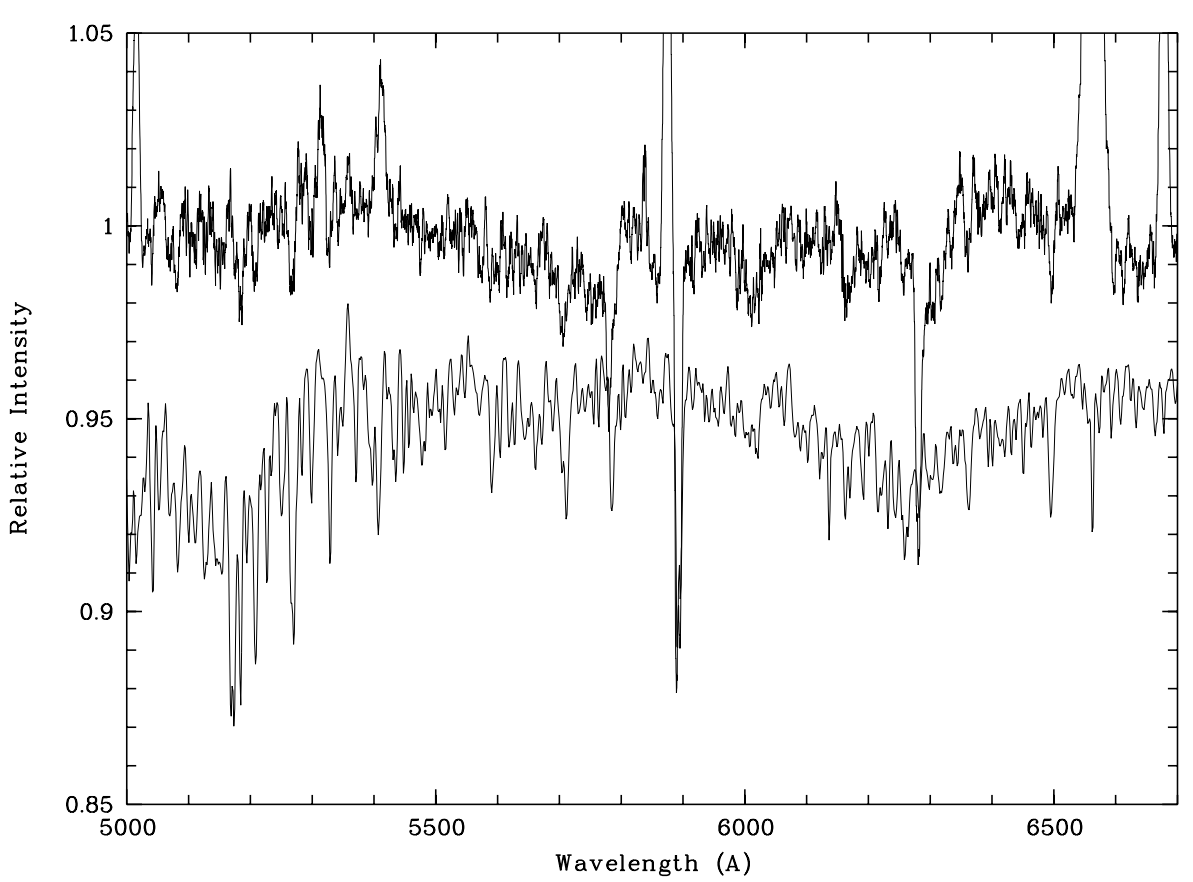

Fig. 4. The red part of the averaged spectrum of 1RXS J154814.5-452845 as observed in 1998 (top) plotted together with a template stellar spectrum of a K4 III star diluted with a constant continuum. Several faint metallic lines visible in the late type spectrum also appear in that of 1RXS J154814.5-452845, revealing the late type secondary (see text).

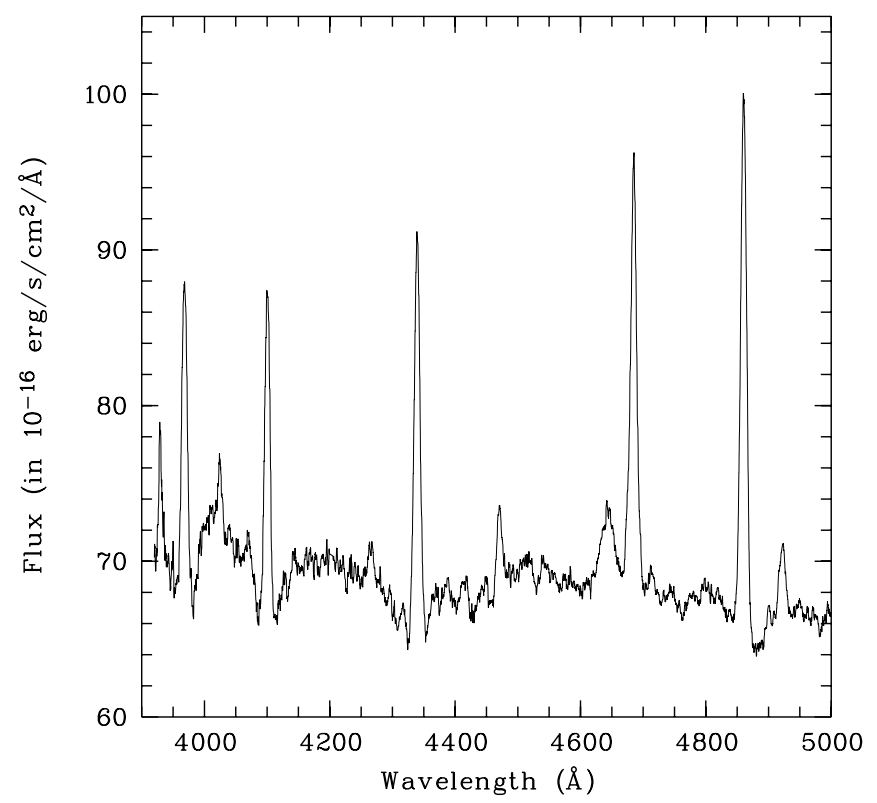

Fig. 5. Blue part of the average 1998 spectrum of 1RXS J154814.5-452845. The broad absorption features underneath the emission spectrum could be the signature of the white dwarf photosphere.

$V$ light curve is 0.036 mag. well in the range of that observed in other intermediate polars (see e.g. Patterson 1994; Welsh \& Martell 1996).

We investigated further the presence of beat frequencies by applying the Lomb-Scargle periodogram to data with the main modulation at $P=692.75$ s removed. The resulting periodogram, plotted in Fig. 10, exhibits an excess power in the interval 120 to 125 cycle/day. The highest peak is at $122.14 \pm 0.14 \mathrm{~d}^{-1}(P=707.39 \pm 0.21 \mathrm{~s})$. At this given frequency, the formal probability of the peak to be real is $1.7 \%$ assuming a white noise local distribution.
The value of the peak frequency agrees very well with that expected for the synodic frequency $\left(f=122.14 \pm 0.21 \mathrm{~d}^{-1}\right)$ computed assuming the best orbital period of $P=9.37 \pm$ $0.69 \mathrm{~h}$, derived from the optical spectroscopy, and a white dwarf spin period of $P=692.75 \pm 0.56 \mathrm{~s}$, derived from the optical photometry. However, an orbital period of $\sim 6.7 \mathrm{~h}$ cannot be strictly ruled out by the observations. At the synodic period the light curve is nearly sinusoidal and has a full amplitude of $0.023 \mathrm{mag}$. The detection of a synodic frequency confirms that the main X-ray and optical period is the true rotation period of the white dwarf.

\section{ROSAT soft $X$-ray observations}

We proposed two observations of 1RXS J154814.5-452845 in the guest observer program of ROSAT using the HRI detector (David et al. 1996). During the first short observation in 1996, Sep. 13 (RH400792, net exposure time of $1.97 \mathrm{ks}$ ) the average count rate was $0.22 \pm 0.01 \mathrm{ctss}^{-1}$. It was dedicated to derive an accurate X-ray position: $\mathrm{RA}=15^{\mathrm{h}} 48^{\mathrm{m}} 14.5^{\mathrm{s}}$, Dec $=-45^{\circ} 28^{\prime} 39^{\prime \prime}$ with an error of $\sim 7^{\prime \prime}$ dominated by systematic boresight uncertainty. After the optical identification as cataclysmic variable and IP candidate a second ROSAT HRI observation (RH300624) was performed in 1998, Sep. 11-14, with a net exposure of $17.89 \mathrm{ks}$. The flux with an average count rate of $0.190 \pm 0.006 \mathrm{cts} \mathrm{s}^{-1}$ was similar to the first HRI observation and also to the survey detection (the HRI to PSPC count rate conversion factor is 3.0 for a hard spectrum).

The second HRI observation, performed near the end of the ROSAT mission, suffered from unstable attitude and no accurate position could be derived. However, the data can be used for a temporal analysis. The HRI light curve $(0.1-2.4 \mathrm{keV})$ shows sinusoidal intensity variations by a factor of about two. A $Z^{2}$ test (Buccheri et al. 1983) yields formally a period of $693.3 \pm 1.1$ s consistent with the 

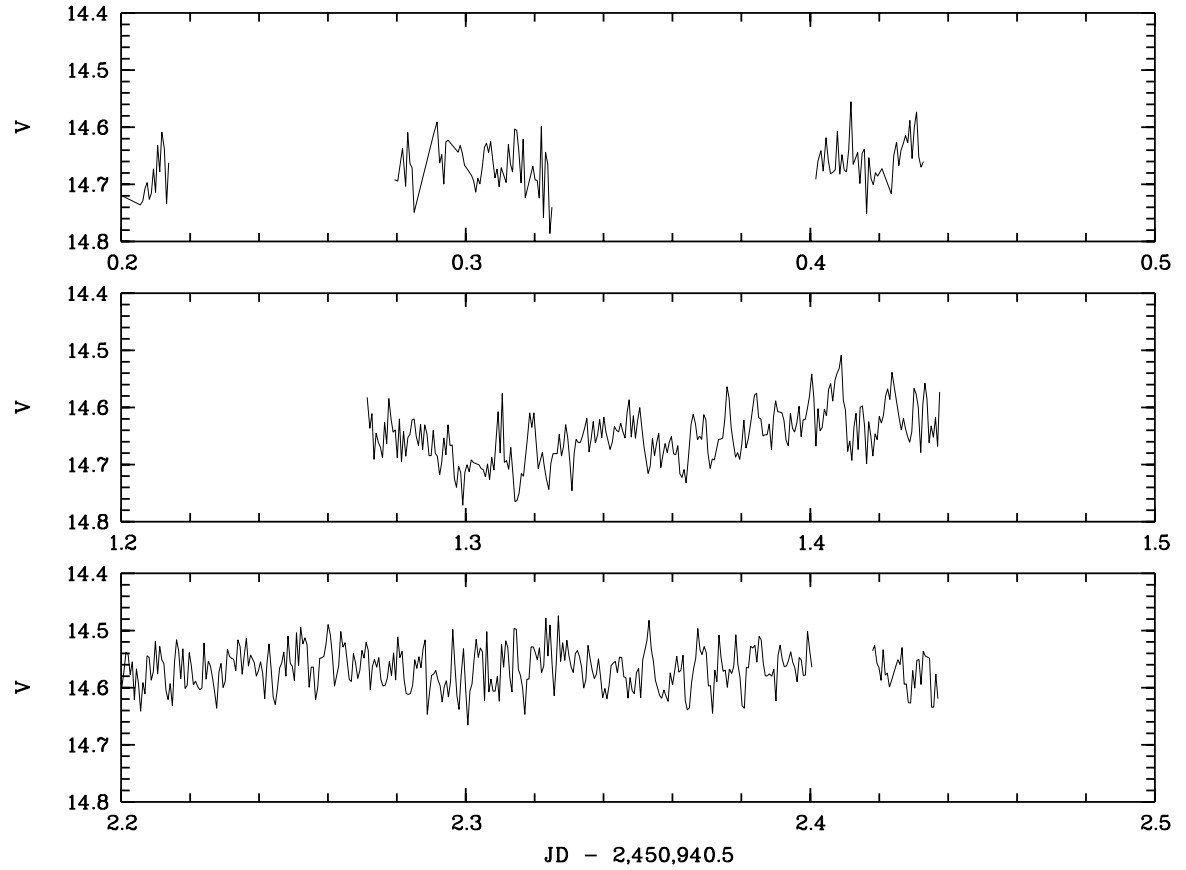

Fig. 6. Optical $V$ band light curve of 1RXS J154814.5-452845 obtained in May 1998 with the ESO-Dutch $0.9 \mathrm{~m}$ telescope.

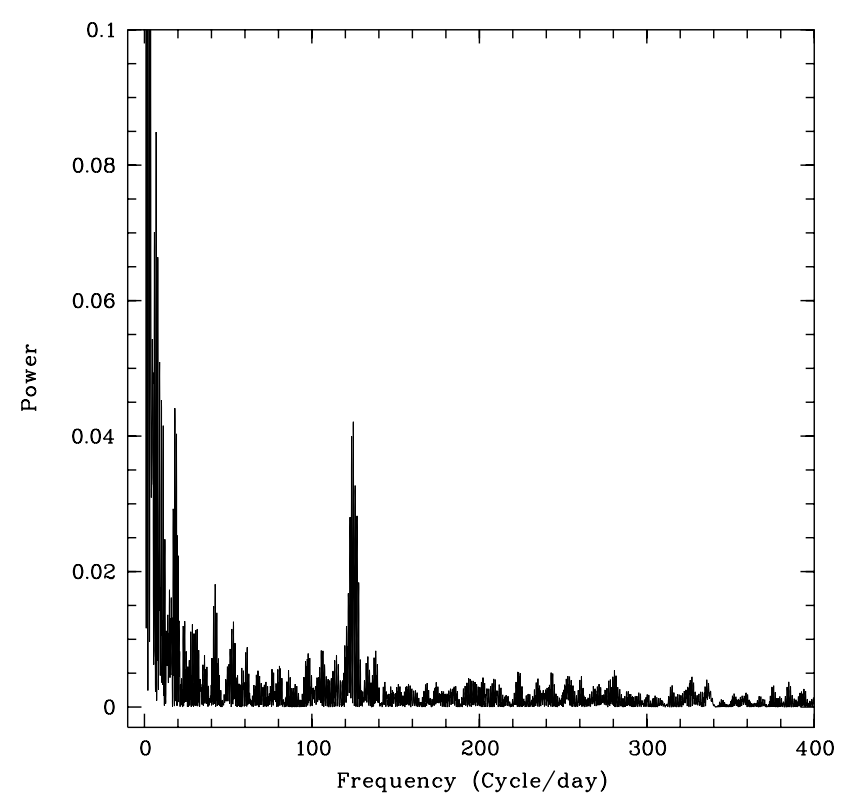

Fig. 7. Periodogram of the $V$ band time series obtained in May 1998 at the ESO-Dutch telescope. A clear periodic signal at $f=124.72 \pm 0.10$ cycle/day $(P=692.75 \pm 0.56 \mathrm{~s})$ is detected. This period is consistent with that seen in the HRI observation.

value derived from the optical observations and confirming 1RXS J154814.5-452845 as IP, however, the large number of short observation intervals typically lasting less than $2000 \mathrm{~s}$ causes many alias peaks. Periods of $682.2 \mathrm{~s}, 627.5 \mathrm{~s}$ and $618.4 \mathrm{~s}$ are of statistically similar significance, but are inconsistent with any of the possible alias periods seen in the optical (Fig. 11). The folded soft X-ray light curve obtained from the HRI data using the $693.3 \mathrm{~s}$ period is presented in Fig. 12.

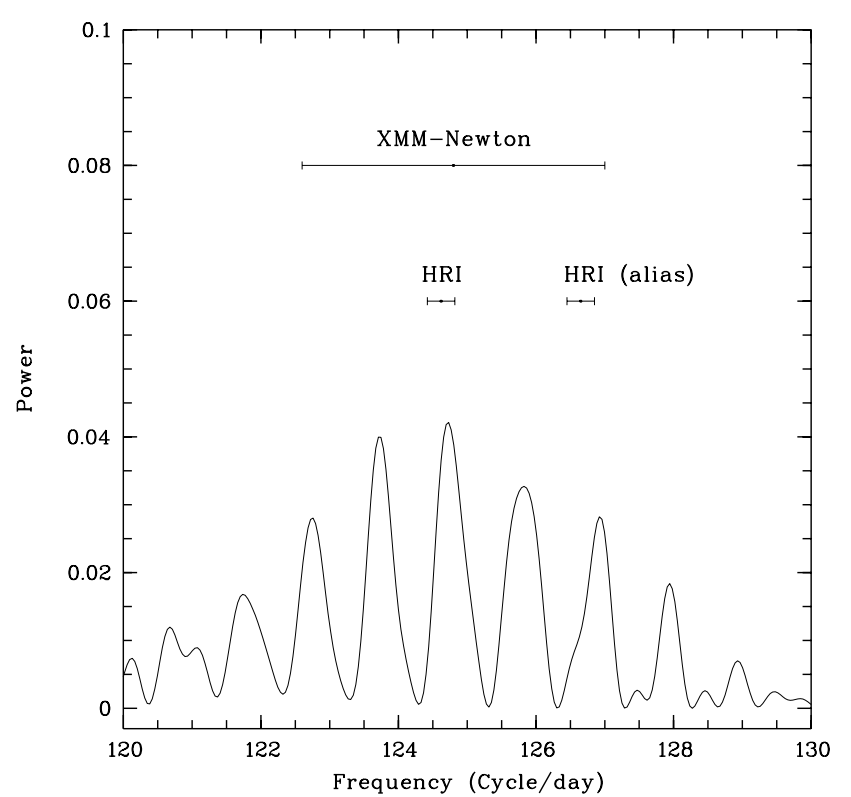

Fig. 8. Detail of the periodogram of the $V$ band time series obtained in May 1998 at the ESO-Dutch telescope. The best optical photometric frequency is consistent with the most likely HRI determination.

\section{XMM-Newton broad band X-ray observations}

XMM-Newton (Jansen et al. 2001) observed 1RXS J154814.5-452845 as part of the telescope scientist guaranteed time during satellite revolution 137. The observation (id 0105460301) on 2000, Sep. 7 lasted for about $20 \mathrm{ks}$ and included the EPIC instruments. The Reflection Grating Systems were switched off due to technical problems and the Optical Monitor was not used due to a bright star in the field. Here we report on the results from the European Photon Imaging Cameras 


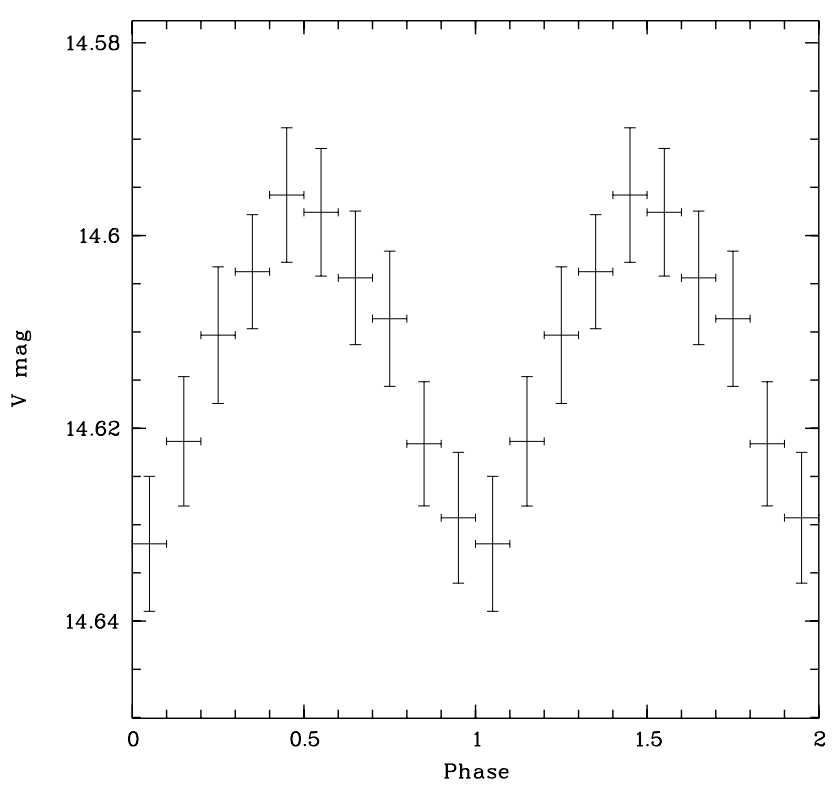

Fig. 9. The $V$ band light curve obtained in 1998 folded with the best period of $P=692.75 \mathrm{~s}$. The mean full amplitude of the modulation is 0.036 mag.

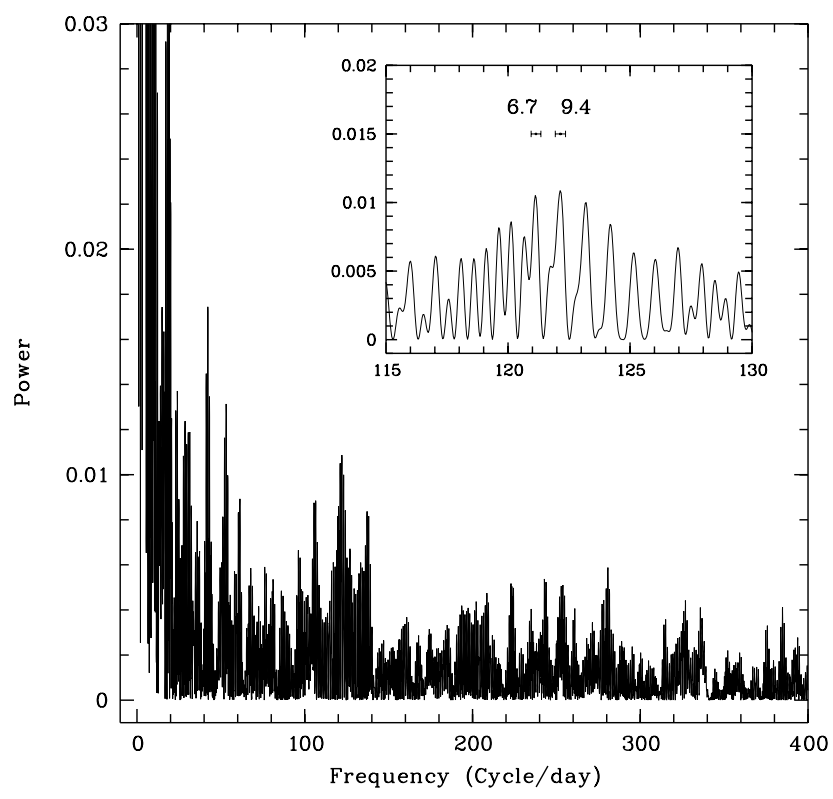

Fig. 10. Periodogram of the $1998 \mathrm{~V}$ band time series demodulated from the main signal at $P=692.75 \pm 0.56$ s. Excess power is seen at the expected position of the synodic frequency. The insert shows were the synodic frequencies should be assuming different orbital periods and the best optically determined white dwarf rotation period. The highest peak of the periodogram corresponds to the best spectroscopic candidate period of $9.4 \mathrm{~h}$.

based on MOS (EPIC-MOS1 and -MOS2, Turner et al. 2001) and PN CCD X-ray detectors (EPIC-PN, Strüder et al. 2001) which are mounted behind the three telescopes (Aschenbach et al. 2000). The background in the EPIC detectors was low during the whole observation with the exception of a short interval of $\sim 400 \mathrm{~s}$ when

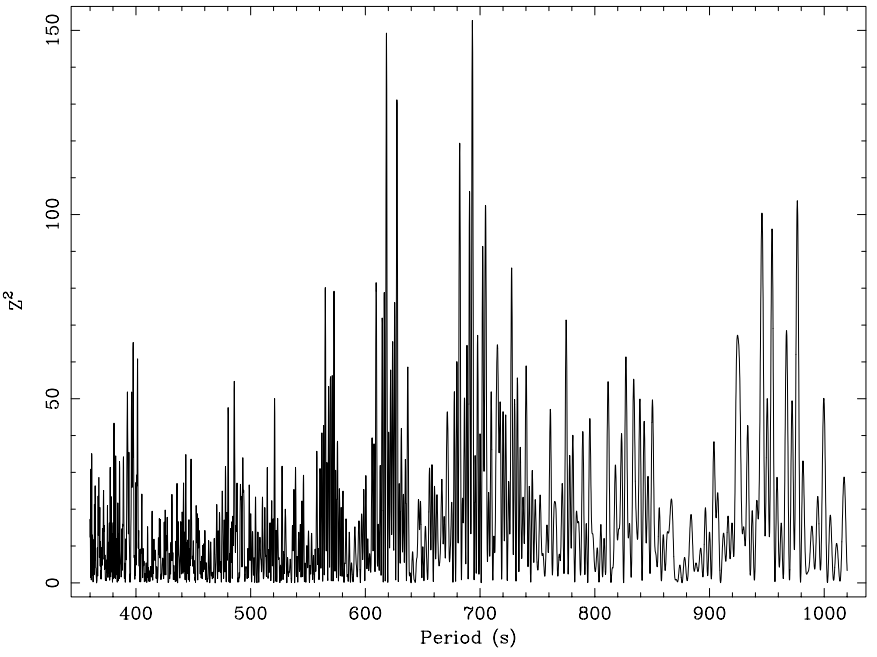

Fig. 11. $\mathrm{Z}^{2}$ test of the ROSAT HRI data.

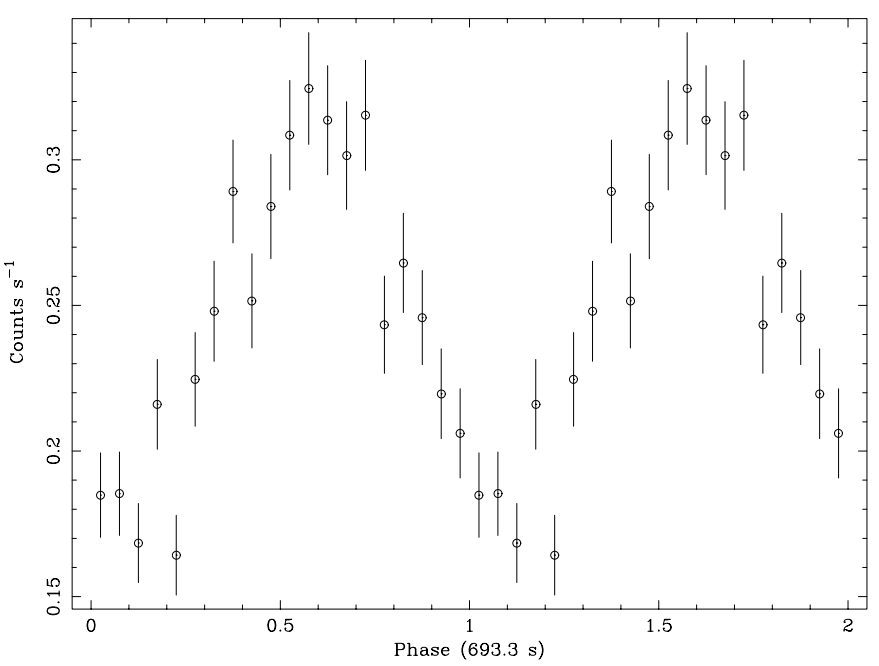

Fig. 12. ROSAT HRI light curve of 1RXS J154814.5-452845 in the $0.1-2.4 \mathrm{keV}$ energy band folded with a period of $693.3 \mathrm{~s}$.

it increased from 1-2 $\mathrm{cts} \mathrm{s}^{-1}$ to about $10 \mathrm{cts} \mathrm{s}^{-1}$ per PN CCD. Source and background events were extracted from circular regions with radius $40^{\prime \prime}$ around the source position and a nearby source-free area in the image.

\subsection{Temporal behaviour}

The light curves in the $0.2-8.0 \mathrm{keV}$ band obtained from $\mathrm{PN}$ and combined MOS data is plotted in Fig. 13. Clearly seen are the periodic $\sim 700 \mathrm{~s}$ intensity variations on top of a more gradual change on a time scale of about $7000 \mathrm{~s}$. A timing analysis using a Fast Fourier Transform (FFT) technique shows a single peak at the spin frequency with no detectable peaks at harmonic or sideband frequencies originating from the beat between spin and binary orbital period. This is consistent with a sinusoidal variation as is seen in the ROSAT HRI data and a long orbital period in excess of the duration of the observation of $\sim 20 \mathrm{ks}$ as is suggested by the optical data. The FFT power spectrum is shown in Fig. 14. There is some signature for a 


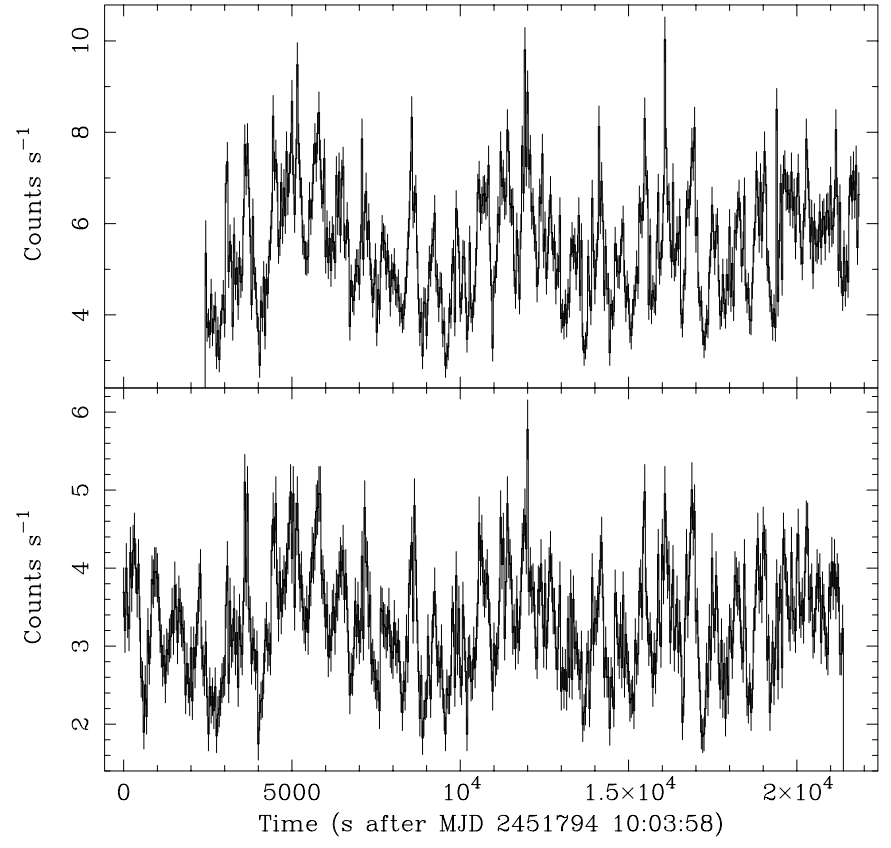

Fig. 13. X-ray broad-band $(0.2-8.0 \mathrm{keV})$ light curves of 1RXS J154814.5-452845 obtained from the XMM-Newton EPIC PN (top) and summed MOS1 and MOS2 (bottom) data with a time binning of $40 \mathrm{~s}$. The average background contribution is $3.6 \times 10^{-2} \mathrm{cts} \mathrm{s}^{-1}$ for PN and $3.3 \times 10^{-2} \mathrm{cts} \mathrm{s}^{-1}$ for MOS1+2.

modulation on a longer time scale of $4000-8000 \mathrm{~s}$ in the power spectrum as was already seen in the light curve. Such a variation might be caused by the binary orbit, but the optical data are more consistent with a longer orbital period. A $\chi^{2}$ folding analysis was used to derive the best value of $692.3 \pm 12 \mathrm{~s}$ for the spin period. For comparison with Fig. 11 the periodogram is shown in Fig. 15. The contiguous XMM-Newton observation leads to a unique identification of the spin period, however, the shorter time base of the observation causes a larger uncertainty.

The EPIC-PN data were folded with a period of $692.3 \mathrm{~s}$ in different energy bands (Fig. 16). The $0.2-2.0 \mathrm{keV}$ profile is consistent with that obtained from the HRI data $(0.1-$ $2.4 \mathrm{keV}$ ). The smaller statistical errors in the PN light curve allow to identify two narrow features. A dip before the pulse maximum (or peak on the increasing flank) and an asymmetric minimum are significant. The intensity dip is more prominent at energies $2.0-8.0 \mathrm{keV}$ while the minimum is more symmetric at high energies. The hardness ratio (ratio of the count rates in the $2.0-8.0 \mathrm{keV}$ to the $0.2-2.0 \mathrm{keV}$ band) shows an increase during intensity minimum. The asymmetry in the soft light curve minimum reflects in a deep dip in the hardness ratio, reaching the low level at intensity maximum. The intensity dip is apparently energy independent and not visible in the hardness ratio. The behaviour of increased hardness during intensity minimum is often observed from IPs and is generally explained in the accretion curtain scenario by increased absorption during pulse minimum caused by the curtain. The sharp and short $(<0.2$ in phase) decline in hardness

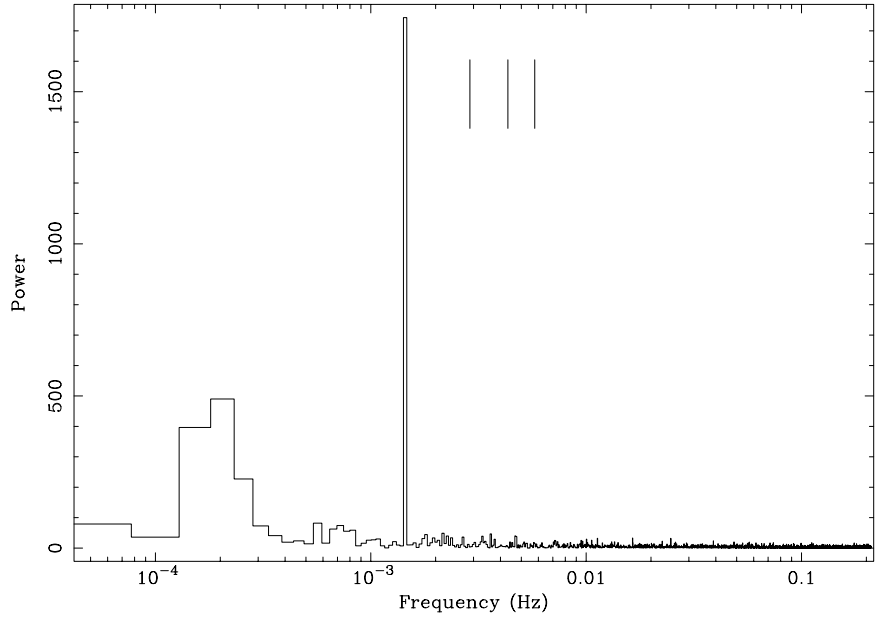

Fig. 14. Power spectrum derived from of the XMM-Newton EPIC-PN data $(0.2-8.0 \mathrm{keV})$. The vertical lines indicate the frequencies of first, second and third harmonics which are not detectable in the power spectrum.

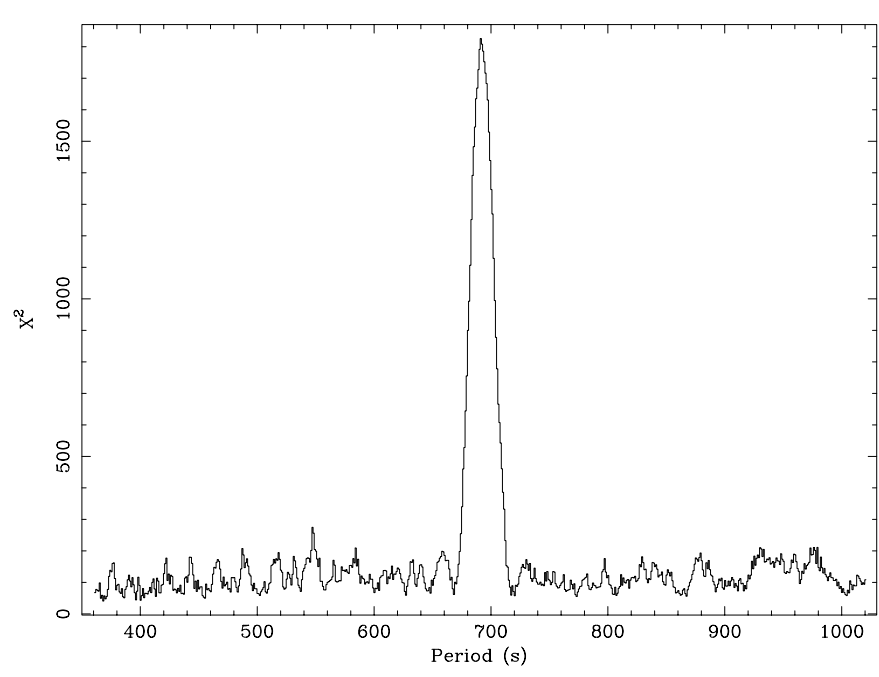

Fig. 15. $\chi^{2}$ periodogram obtained from the XMM-Newton EPIC-PN data $(0.2-8.0 \mathrm{keV})$.

ratio near intensity minimum then suggests that the line of sight at this phase is nearly parallel to the curtain, allowing an almost free view down to the white dwarf surface. As the white dwarf rotates the curtain quickly intersects the line of sight causing increased absorption. The hardness ratio dip, the sinusoidal pulse variation with a single peak and the narrow optical emission lines suggest that we see emission from one pole only (if we indeed look down nearly parallel to the curtain the opposite pole in a dipole field should be hidden by the white dwarf at this spin phase). One should mention here that EX Hya also shows sinusoidal pulse variations while we see emission from both poles as indicated by, however, broad optical emission lines (Hellier et al. 1987). 


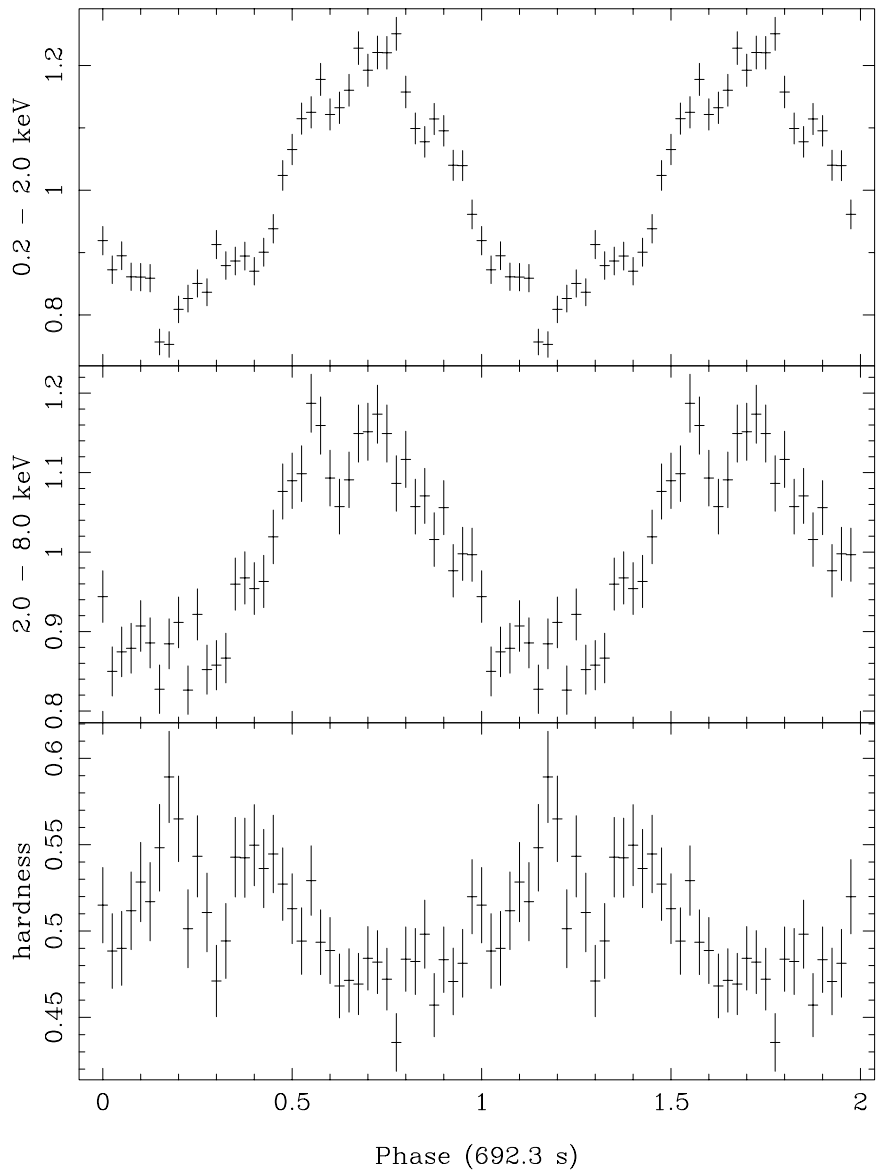

Fig. 16. Folded light curves of 1RXS J154814.5-452845 derived from XMM EPIC-PN data in two energy bands together with their hardness ratio $(2.0-8.0 \mathrm{keV} / 0.2-2.0 \mathrm{keV})$. The folded light curves are normalized to the average count rate $\left(3.67 \mathrm{cts} \mathrm{s}^{-1}\right.$ in the $0.2-2.0 \mathrm{keV}$ band and $1.84 \mathrm{cts} \mathrm{s}^{-1}$ in the $2.0-8.0 \mathrm{keV}$ band and are plotted versus an arbitrary phase.

\subsection{The spin-phase averaged energy spectrum}

To further investigate the emission properties of 1RXS J154814.5-452845 spin-averaged energy spectra from the EPIC detectors were accumulated. From the PN data single- (pattern 0) and double-pixel (pattern 14) events were selected into two separate spectra to utilize the better energy resolution for single-pixel events. For the two MOS spectra pattern 0-12 events were used. The four spectra were simultaneously fit with the same spectral model only allowing different normalizations between the two MOS and the PN spectra to account for possible inter-calibration problems in absolute fluxes and the different temporal coverage of the instruments (Fig. 13). Spectral response files provided by the hardware teams were used (epn_ff20_sY9_medium.rmf and epn_ff20_dY9_medium.rmf for PN single and double events released in March 2001; m1_medv9q19t5r4_all_15.rsp and m2_medv9q19t5r4_all_15.rsp for MOS released in Feb. 2001). In the past various spectral emission and absorption components were required to fit X-ray spectra of IPs. These include emission from hot thin plasma and soft blackbody radiation (dominating the spectrum of soft IPs in the ROSAT band). In the following different combinations of these models are compared with the observed EPIC spectra. In all models absorption partially covering the X-ray source is applied and an iron fluorescence line is included.

\subsubsection{Emission from thin plasma with constant temperature}

A spectral model consisting of hot thermal bremsstrahlung suffering absorption by matter partially covering the X-ray source together with an iron line complex (a fluorescent line from nearly neutral iron and two lines from FeXXV and Fe XXVI) as it was representing X-ray spectra from classical IPs in the past gives no acceptable fit to the EPIC spectra of 1RXS J154814.5-452845. We used the MEKAL model (Mewe et al. 1985) for thermal plasma emission from XSPEC 11.0.1 to account for the bremsstrahlung component and the two lines from highly ionized Fe (model A, see Table 3 ). This resulted in a reduced $\chi^{2}$ of 3.5 with a large excess flux below $0.8 \mathrm{keV}$.

\subsubsection{Thin plasma emission plus blackbody component}

Improved fit results were obtained by adding a blackbody component to account for the low-energy excess (model B), improving the reduced $\chi^{2}$ to 1.30. In this model the low absorption component of the partial absorber was also applied to the blackbody emission. Around $1 \mathrm{keV}$ the fit is still not acceptable, again showing excess flux. This excess indicates iron $L$ emission as is also seen in high resolution spectra from the IP RE $0751+14=$ PQ Gem (Burwitz et al. 2001).

\subsubsection{Two-temperature thin plasma emission plus blackbody component}

For iron $L$ emission a lower temperature of the emitting matter is required and we added another MEKAL component (soft thermal $k T_{\mathrm{MK}}^{\mathrm{s}}$ component in contrast to the hard thermal $k T_{\mathrm{MK}}^{\mathrm{h}}$ component) to the low absorbed part of the model spectrum (model C). This gives an acceptable fit over the whole energy band with reduced $\chi^{2}$ of 1.26 for 2113 d.o.f.

\subsubsection{Multi-temperature thin plasma emission plus blackbody component}

The co-existence of soft $(1 \mathrm{keV})$ and hard $(14 \mathrm{keV})$ thermal emission indicates that we actually observe a wide range of temperatures. A multi-temperature model was used by Done \& Magdziarz (1998) to fit ASCA and Ginga spectra from BY Cam. Such a model is available in the XSPEC package, based on the MEKAL model with 
Table 3. Spectral fit results for models A to E.

\begin{tabular}{|c|c|c|c|c|c|c|c|c|c|c|c|}
\hline & \multicolumn{2}{|c|}{ high absorption } & \multicolumn{5}{|c|}{ low absorption } & \multirow{2}{*}{$\begin{array}{l}\text { temp. } \\
\text { distr. } \alpha\end{array}$} & \multirow{2}{*}{$\begin{array}{l}\text { metal } \\
\text { abund. }\end{array}$} & \multirow[t]{2}{*}{$\chi^{2}$} & \multirow[t]{2}{*}{ d.o.f. ${ }^{a}$} \\
\hline & $\begin{array}{c}N_{\mathrm{H}} \\
{\left[10^{23} \mathrm{~cm}^{-2}\right]}\end{array}$ & $\begin{array}{r}k T_{\mathrm{MK}}^{\mathrm{h}} \\
{[\mathrm{keV}]} \\
\end{array}$ & $\begin{array}{c}N_{\mathrm{H}} \\
{\left[10^{21} \mathrm{~cm}^{-2}\right]}\end{array}$ & $\begin{array}{c}k T_{\mathrm{MK}}^{\mathrm{h} b} \\
{[\mathrm{keV}]} \\
\end{array}$ & $\begin{array}{c}k T_{\mathrm{bb}} \\
{[\mathrm{eV}]} \\
\end{array}$ & $\begin{array}{r}k T_{\mathrm{MK}}^{\mathrm{s}} \\
{[\mathrm{keV}]} \\
\end{array}$ & $\begin{array}{c}\mathrm{Fe}_{\text {eqw }}^{6.4} \\
{[\mathrm{eV}]} \\
\end{array}$ & & & & \\
\hline $\mathrm{A}$ & 1.53 & 10.0 & 0.1 & 10.0 & - & - & 286 & - & 0.34 & 7444 & 2117 \\
\hline B & $1.35 \pm 0.11$ & $13.7 \pm 0.8$ & $1.23 \pm 0.06$ & 13.7 & $95 \pm 2$ & - & $272 \pm 27$ & - & $0.50 \pm 0.05$ & 2748 & 2115 \\
\hline $\mathrm{C}$ & $1.21 \pm 0.11$ & $14.3 \pm 0.8$ & $1.42 \pm 0.07$ & 14.3 & $89 \pm 2$ & $1.03 \pm 0.07$ & $282 \pm 29$ & - & $0.53 \pm 0.05$ & 2657 & 2113 \\
\hline $\mathrm{D}$ & $0.97 \pm 0.10$ & $59 \pm 10^{c}$ & $1.39 \pm 0.05$ & 59 & $88 \pm 2$ & - & $253 \pm 24$ & $1.0^{d}$ & $0.98_{-0.12}^{+0.21}$ & 2552 & 2115 \\
\hline $\mathrm{E}$ & $0.95 \pm 0.08$ & $60^{c, d}$ & $1.47 \pm 0.08$ & 60 & $86 \pm 2$ & - & $258 \pm 26$ & $0.90 \pm 0.05$ & $0.90_{-0.06}^{+0.10}$ & 2544 & 2115 \\
\hline
\end{tabular}

For model A no errors were determined because of the unacceptable fit.

${ }^{a}$ Degrees of freedom.

${ }^{b}$ Parameter linked to temperature of high absorption MEKAL component.

${ }^{c}$ Maximum temperature $T_{\max }$ in multi-temperature models.

${ }^{d}$ Fixed parameter.

emission measures which follow a power-law in temperature (the emission measure from temperature $T$ is proportional to $\left.\left(T / T_{\max }\right)^{\alpha}\right)$. To fit this model we replaced the two MEKAL components by the multi-temperature component, suffering absorption again by matter partially covering the X-ray source. As first approach we fixed the parameter $\alpha$ at 1.0 (model D in Table 3). The fit further improved to a reduced $\chi^{2}$ of 1.21 for 2115 d.o.f. (the $\chi^{2}$ decreased by $\sim 100$ although the number of free parameters was reduced by 2). Alternatively allowing $\alpha$ as free fit parameter the maximum temperature is not constrained and was therefore fixed at $60 \mathrm{keV}$, the value derived with fixed $\alpha$. This model ( $\mathrm{E}$ in Table 3 ) finally yields the best fit (reduced $\chi^{2}$ of 1.21 ) although statistically not different from model D. The spectra (PN singles+doubles, MOS1 and MOS2) are shown in Fig. 17 together with the best fit model E.

As derived from the normalizations of the differently absorbed multi-temperature emission components, the high column density matter covers the plasma by $47 \%$ while $53 \%$ are absorbed with a much reduced column density. The slope of the optical spectrum indicates that the column density of the low absorption component, which corresponds to $E(B-V) \sim 0.25$, could entirely be of interstellar origin. The flux determined from the spectra in the $0.2-10.0 \mathrm{keV}$ band was $2.28 \times 10^{-11} \mathrm{erg} \mathrm{cm}^{-2} \mathrm{~s}^{-1}$, $2.34 \times 10^{-11} \mathrm{erg} \mathrm{cm}^{-2} \mathrm{~s}^{-1}$ and $2.30 \times 10^{-11} \mathrm{erg} \mathrm{cm}^{-2} \mathrm{~s}^{-1}$ for MOS1, MOS2 and PN respectively. PN single and double spectrum were forced to have the same normalization in the fit and therefore yield the same flux. The fluxes derived from the three detectors agree remarkably well, it should be noted however that the used data does not cover identical time intervals. The spectra are not corrected for point spread function losses outside the source extraction radius. The used radius of $40^{\prime \prime}$ should encompass $93 \%$ of the flux (Aschenbach et al. 2000) with little energy dependence. The intrinsic $0.2-10.0 \mathrm{keV}$ flux (with absorption set to 0 ) of the multi-temperature emission component is $3.3 \times 10^{-11} \mathrm{erg} \mathrm{cm}^{-2} \mathrm{~s}^{-1}$ and the bolometric blackbody flux is $1.5 \times 10^{-11} \mathrm{erg} \mathrm{cm}^{-2} \mathrm{~s}^{-1}$, yielding a ratio for blackbody to plasma emission of $\sim 46 \%$.

The spectra around the $\mathrm{FeK}$ emission line complex are shown enlarged in Fig. 18. Iron fluorescence, Fe XXV and Fe XXVI lines are clearly resolved in the PN singleevents and MOS spectra. The fluorescence line energy derived from the fits is $6.396 \pm 0.007 \mathrm{keV}$ (models C-E). The equivalent width $\left(\mathrm{Fe}_{\text {eqw }}^{6.4}\right)$ is listed in Table 3. The Fe XXV and Fe XXVI line intensities require an underabundance of iron in models A-C. We therefore allowed to vary the metal abundance in the MEKAL components, but linked all metal abundances in all MEKAL components to one single free parameter, which is mainly determined by the iron lines. The best fit values for the metal abundance varies considerably between models B$\mathrm{C}$ with $\sim 0.5$ solar and the multi-temperature models $\mathrm{D}$ and $\mathrm{E}$ where it is consistent with the solar value (Table 3 ). For the equivalent hydrogen densities given in Table 3 solar abundances in the absorbing matter are assumed. At least for the high absorption column, which is intrinsic to the 1RXS J154814.5-452845 system, reduced metal abundances by a factor of two would increase the hydrogen column density by the same factor.

There is indication for a stronger Fe XXV line than the model predicts. Producing more He-like iron emission relative to $\mathrm{H}$-like iron emission requires a lower temperature than the overall fit (which includes the bremsstrahlung continuum) predicts. Fits to RXTE and BeppoSAX spectra of IPs extending to energies up to nearly $100 \mathrm{keV}$ yield reduced temperatures when a hard emission component as arises from reflection on the white dwarf surface is included in the spectral model (de Martino et al. 2001).

\subsection{Phase resolved spectral analysis}

Spectra were accumulated using events from spin phase 0.45-0.95 (spin maximum) and 0.95-0.45 (spin minimum, see Fig. 16). Subtracting the spin minimum from spin maximum spectrum shows an increasing count rate from 
RXJ1548.2-4528

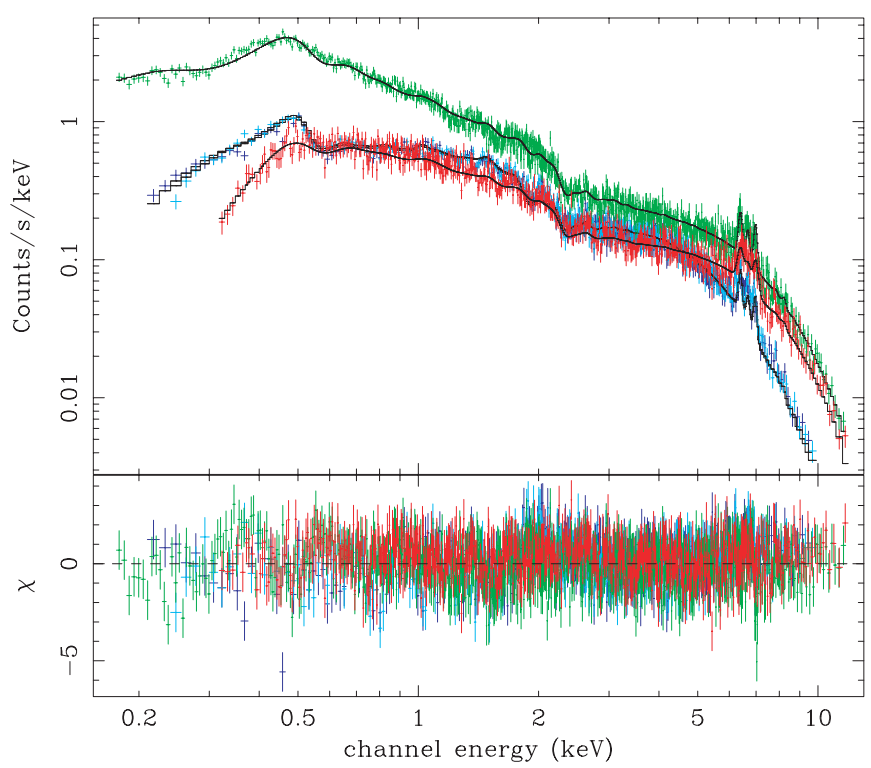

Fig. 17. EPIC MOS (blue and light blue) and PN (singlepixel events green, double-pixel events red) spectra of 1RXS J154814.5-452845 together with the best fit model E (see text). The bottom panel shows the residuals. Somewhat larger deviations near $0.5 \mathrm{keV}$ and $2.2 \mathrm{keV}$ may be caused by calibration uncertainties around instrumental absorption edges.

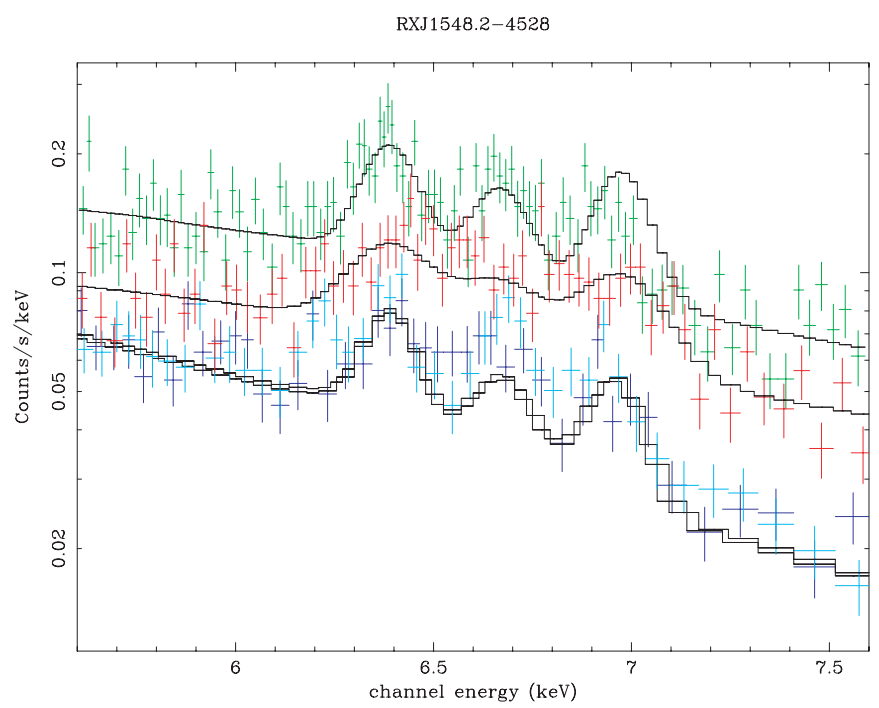

Fig. 18. Enlarged part of Fig. 17 showing the Fe line complex in the EPIC spectra of 1RXS J154814.5-452845. The colour coding is as in Fig. 17.

$0.2 \mathrm{keV}$ up to a maximum around $0.5 \mathrm{keV}$ and then a decline down to $2 \mathrm{keV}$. This suggests that mainly the two soft components (blackbody and $1 \mathrm{keV}$ MEKAL in model $\mathrm{C}$ ) change their intensity during the white dwarf spin revolution. In particular the decrease of the difference spectrum towards low energies indicates that absorption changes play a minor role.

A fit to spin minimum and maximum spectra using models $\mathrm{C}$ and $\mathrm{E}$ shows that temperatures and element abundances do not change. Therefore, these parameters
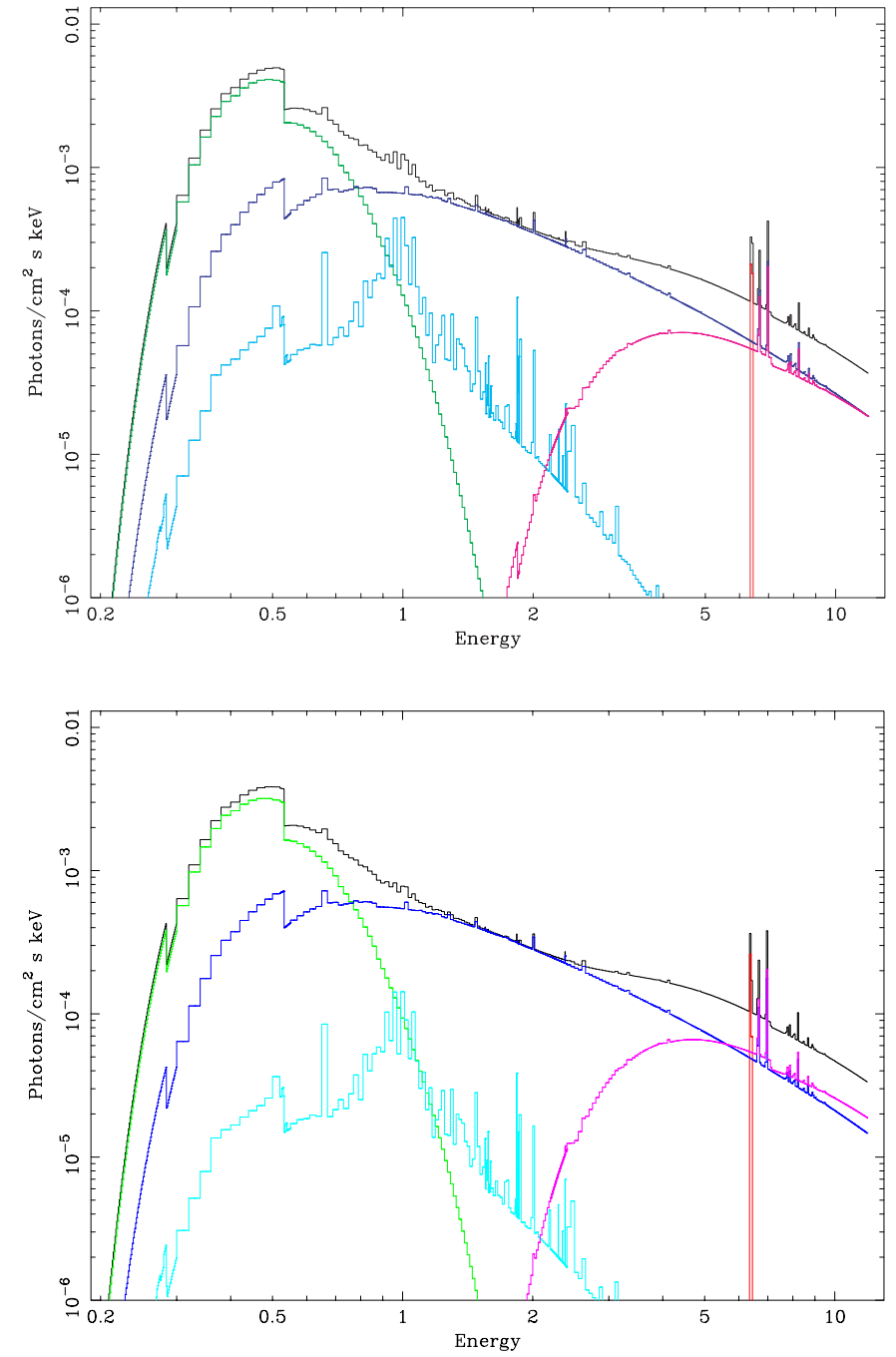

Fig. 19. The spectral model components (model $\mathrm{C}$ described in the text) compared for spin minimum (bottom) and maximum (top). Most of the intensity variations are caused by the soft thermal emission (light blue). This emission component shows an Fe-L line complex near $1 \mathrm{keV}$ and is reduced by a factor of $\sim 3$ during spin minimum.

were fixed at the values derived from the spin averaged spectra. The normalizations and column densities derived from the fits using models $\mathrm{C}$ and $\mathrm{E}$ are compared for spin maximum and minimum spectra (for the case of $\mathrm{PN}$ ) in Table 4. As suggested by the difference spectrum, the column density of the high absorption component shows only a marginal change between spin minimum and maximum. The parameters for absorption and temperature agree within the errors, although there is an indication for some $N_{\mathrm{H}}$ decrease during spin maximum. Also the $N_{\mathrm{H}}$ values of the low absorption components are consistent within errors. From the low absorption components the soft thermal component (model C) shows the strongest intensity increase during spin maximum of at least a factor of 2.0 while the blackbody and the hard thermal components vary at most by factors 1.7 and 1.3, respectively. This is illustrated in Fig. 19 where the model components 
Table 4. Intensity variations in the spectral components (temperatures and element abundances are fixed at values derived from spin averaged spectra).

\begin{tabular}{|c|c|c|c|c|c|c|c|c|}
\hline \multirow{2}{*}{$\begin{array}{c}\text { Model/ } \\
\text { Spin phase }\end{array}$} & \multicolumn{2}{|c|}{ high absorption } & \multicolumn{5}{|c|}{ low absorption } & \multirow{2}{*}{$\begin{array}{l}\text { temp. } \\
\text { distr. } \alpha\end{array}$} \\
\hline & $\begin{array}{c}N_{\mathrm{H}} \\
{\left[10^{23} \mathrm{~cm}^{-2}\right]}\end{array}$ & $\begin{array}{c}\operatorname{norm}_{\mathrm{MK}}^{\mathrm{h}} \\
{\left[10^{-3}\right]}\end{array}$ & $\begin{array}{c}N_{\mathrm{H}} \\
{\left[10^{21} \mathrm{~cm}^{-2}\right]}\end{array}$ & $\begin{array}{c}\operatorname{norm}_{\mathrm{MK}}^{\mathrm{h}} \\
{\left[10^{-3}\right]}\end{array}$ & $\begin{array}{c}\operatorname{norm}_{\mathrm{bb}} \\
{\left[10^{-4}\right]}\end{array}$ & $\begin{array}{c}\operatorname{norm}_{\mathrm{MK}}^{\mathrm{s}} \\
{\left[10^{-4}\right]}\end{array}$ & $\begin{array}{c}\mathrm{Fe}_{\text {eqw }}^{6.4} \\
{[\mathrm{eV}]}\end{array}$ & \\
\hline C/Min. & $1.29 \pm 0.15$ & $4.76 \pm 0.27$ & $1.41 \pm 0.08$ & $3.45 \pm 0.09$ & $0.74 \pm 0.07$ & $0.98 \pm 0.35$ & $302 \pm 53$ & - \\
\hline C/Max. & $1.07 \pm 0.15$ & $4.59 \pm 0.26$ & $1.53 \pm 0.08$ & $4.32 \pm 0.11$ & $1.05 \pm 0.08$ & $3.12 \pm 0.44$ & $287 \pm 47$ & - \\
\hline E/Min. & $1.01 \pm 0.18$ & $7.55 \pm 0.45$ & $1.49 \pm 0.08$ & $7.33 \pm 0.54$ & $0.88 \pm 0.09$ & - & $277 \pm 53$ & $0.95 \pm 0.06$ \\
\hline E/Max. & $0.78 \pm 0.13$ & $6.44 \pm 0.37$ & $1.53 \pm 0.08$ & $7.67 \pm 0.48$ & $1.12 \pm 0.11$ & - & $265 \pm 41$ & $0.76 \pm 0.04$ \\
\hline
\end{tabular}

are drawn for spin minimum and maximum. In model $\mathrm{E}$ the intensity increase of the soft thermal emission is reflected in a decrease of the power-law index $\alpha$ of the temperature distribution.

The equivalent width of the Fe fluorescence line varies very little between spin minimum and maximum. A small decrease during spin maximum is insignificant given the large errors. If the fluorescence line would mainly originate on the white dwarf surface a correlation of its equivalent width with other emission from the surface may be expected. However, the blackbody emission increases during spin maximum suggesting that a large part of the line intensity is probably produced in the cold pre-shock matter of the accretion curtain which strongly absorbs the hard X-ray emission. In this case little changes in the equivalent width are expected as long as the line emitting volume remains visible to the observer. In contrast to 1RXS J154814.5-452845 de Martino et al. (2001) detect variations in the equivalent width of the Fe fluorescence line from RX J0028.8+5917 during a short interval in spin phase (of duration 0.2) which we may miss due to our coarse phase resolution.

Together with the dip in hardness ratios around spin minimum the spectral variations seen between spin minimum and maximum suggest that we are viewing the white dwarf nearly pole-on. If the soft thermal component originates in the accretion curtain below the shock region, the variation in its intensity would then be caused mainly by the varying aspect angle under which the curtain is observed during the spin revolution and suggests beaming of the radiation preferentially out of the sides of the accretion curtains. This is consistent with the decrease in covering fraction of the high column density absorber from $51 \%$ at spin minimum to $46 \%$ at maximum as calculated from the norm $_{\mathrm{MK}}^{\mathrm{h}}$ values for model $\mathrm{E}$ given in Table 4 .

\section{Discussion}

Our extensive optical and X-ray observations establish 1RXS J154814.5-452845 as an intermediate polar with rather unique properties. Optical spectroscopy and photometry indicate an orbital period of $6.72 \mathrm{~h}$ or $9.37 \mathrm{~h}$, probably together with GK Per (47.9 h), V 1062 Tauri
(9.96 h) and AE Aqr (9.88 h) among the longest known from IPs. The optical spectrum is strikingly similar to that of V 709 Cas (Bonnet-Bidaud et al. 2001) with broad absorption lines at $\mathrm{H} \beta$ and higher order Balmer lines. Bonnet-Bidaud et al. (2001) argue that these are produced in the non heated part of the white dwarf atmosphere. Therefore, 1RXS J154814.5-452845 may be also a rare case where the three constituents of the system, hot white dwarf, accretion disc and mass donor star can be detected altogether at optical wavelength. This peculiarity opens prospects for an accurate determination of system parameters.

All X-ray observations of 1RXS J154814.5-452845 found the source at a similar X-ray flux level. Using the spectral model parameters derived from the EPIC spectra expected ROSAT PSPC and HRI count rates of 0.400 cts s$^{-1}$ and 0.146 cts s$^{-1}$, respectively, were simulated. This shows that the source was brightest during the Sep. 1996 observation, a factor of 1.5 brighter than in Sep. 2000 when it was at the lowest observed intensity level.

To fit the X-ray spectra of 1RXS J154814.5-452845 as obtained by the EPIC instruments $(0.15-12 \mathrm{keV})$ a complex model is required. Thermal emission from a hot multi-temperature plasma in collisional ionization equilibrium together with absorption by matter partially covering the hard X-ray source represents the spectrum above $1 \mathrm{keV}$. An approach with a two-temperature model suggests that we see a wide range of temperatures covering at least 1 to $14 \mathrm{keV}$. High resolution spectra of magnetic $\mathrm{CVs}$ are consistent with a continuous temperature distribution (Mauche 2001; Burwitz et al. 2001) as is expected from the cooling matter in the post-shock accretion column. The fit of a model with a continuous temperature distribution results in a maximum plasma temperature of $60 \pm 10 \mathrm{keV}$, relatively unconstrained by the limited bandpass of the EPIC instruments. The temperature of the post-shock region expected from the simple shock model $k T=3 / 8 \mathrm{G} \mu \mathrm{m}_{\mathrm{H}} M_{\mathrm{wd}} / R_{\mathrm{wd}}$ with the mean molecular weight $\mu$ and the hydrogen mass $\mathrm{m}_{\mathrm{H}}$, for a $1 M_{\odot}$ white dwarf with a radius of $0.008 R_{\odot}$ (for a mass radius relation see e.g. Panei et al. 2000) is $\sim 60 \mathrm{keV}$. However this may be regarded only as upper limit because spectral 
models including other hard spectral components result in decreased shock temperatures (Beardmore et al. 2000). Fitting Ginga LAC spectra of V1223 Sgr with and without taking into account reflection of the hard bremsstrahlung from the white dwarf surface these authors found shock temperatures of $30_{-4}^{+12} \mathrm{keV}$ and $57 \mathrm{keV}$, respectively. Reducing the shock temperature to $30 \mathrm{keV}$ translates to a white dwarf mass of $\sim 0.7 M_{\odot}$. A more reliable shock temperature and from this a better constrained estimate for the white dwarf mass requires the measurement of the hard X-ray spectrum at energies above $10 \mathrm{keV}$.

At energies below $1 \mathrm{keV}$ a blackbody component with a temperature $\mathrm{kT}$ of $86 \mathrm{eV}$ is needed to fit the EPIC spectra. The bolometric blackbody flux is $\sim 46 \%$ of the intrinsic $0.2-10 \mathrm{keV}$ hot plasma emission. This fraction is lower than that observed from AMHer systems and soft IPs, where the blackbody flux exceeds the bremsstrahlung emission. A correlation of the flux ratio with the magnetic field strength at the main accreting pole suggests increasing suppression of bremsstrahlung in strong magnetic fields (Beuermann \& Burwitz 1995). The low flux ratio derived for 1RXS J154814.5-452845 is consistent with a magnetic field strength below $10 \mathrm{MG}$ as generally anticipated for classical IPs. The temperature of $86 \mathrm{eV}$ is higher than that observed from AMHer systems and soft IPs which show typical values of $20-40 \mathrm{eV}$ (e.g. Matt et al. 2000) and 50-60 eV (Duck et al. 1994; Haberl et al. 1994), respectively. Models for the accretion process onto magnetic white dwarfs predict that the effective temperature decreases with increasing size of the area covered by accretion on the white dwarf (King \& Lasota 1990). The lack of soft X-ray emission from IPs supported this view and suggested temperatures too low for soft X-ray emission to be detected. The case of 1RXS J154814.5-452845 and in this sense also the soft IPs show an opposite behaviour: the temperature increases from AMHer systems via soft IPs to 1RXS J154814.5-452845, i.e. from high magnetic field strength (small accretion area) to low magnetic field strength (large accretion area). This suggests that either 1RXS J154814.5-452845 is a very peculiar case or that the temperature characterizing the soft emission is determined mainly by other accretion parameters like the mass accretion rate or mass and radius of the white dwarf. Hellier (1999) also discussed that blobby accretion im AMHer systems may lead to a larger footprint on the white dwarf, while in IPs accretion of more homogenous matter from the accretion disc probably results in a narrow radial confinement of the accretion curtain, compensating for the larger range in azimuth.

For an absorbing column density of $10^{23} \mathrm{~cm}^{-2}$ the expected equivalent width of the Fe fluorescent line should be of the order of $\sim 100 \mathrm{eV}$ (Inoue 1985). It is however not clear if the spherical geometry of the absorbing matter around the early type star in a high mass X-ray binary as used by Inoue (1985) can be transfered to the situation we have in magnetic CVs with a more cylindrical geometry of the accretion stream. From a tall cylinder with the line of sight along the cylinder axis high absorption is ex- pected but the cylinder contains relatively little matter to produce the fluorescence line. In a flat cylinder the absorption is low but the fluorescence line stronger. In the accretion curtain scenario of IPs the geometry may be closer to the latter case and indeed be the main reason for the high equivalent width of the Fe fluorescence line observed in 1RXS J154814.5-452845 and other IPs. To estimate the Fe line contribution from reflection by cold matter on the white dwarf surface a more detailed modeling of the $\mathrm{Fe}$ line production in the accreting matter is required and arguments based on the results of Inoue (1985) sometimes used in the literature should be taken with caution.

The spectral analysis of 1RXS J154814.5-452845 has shown a strong model dependence of the derived metal abundances. While one- or two-temperature plasma emission models result in abundances of $50 \%$ solar, the models with continuous temperature distribution yield values compatible with solar elemental abundances. This is likely caused by insufficient spectral resolution in particular at low energies which does not allow to properly disentangle line from continuum emission. To constrain elemental abundances of the accreted matter high resolution spectra obtained from grating spectrometers are required and values derived from broad-band, medium resolution spectra should be treated with caution.

\section{Conclusions}

The follow-up observations of 1RXS J154814.5-452845 at optical and X-ray wavelength have revealed a new intermediate polar with interesting properties. The pulsations with a period of $693 \mathrm{~s}$ most likely reflect the rotation period of the white dwarf and the optical data suggest a long orbital period of more than 6 hours. Optical spectra are consistent with a late type companion of spectral type $\mathrm{K}$ and the presence of broad absorption features underneath the Balmer emission lines could be the signature of the white dwarf photosphere, similar to the classical intermediate polar V 709 Cas. The improved spectral resolution of the XMM-Newton EPIC instruments together with the large collecting area of the X-ray mirrors allowed a detailed study of the broad band $0.1-12 \mathrm{keV}$ spectrum. The spectrum can be modeled by multi-temperature emission from hot plasma originating in the post-shock accretion region with a maximum temperature of $\sim 60 \mathrm{keV}$, an iron fluorescence line with an equivalent width of $\sim 260 \mathrm{eV}$ and a $86 \mathrm{eV}$ blackbody component. About $47 \%$ of the hard emission is absorbed by matter partially covering the X-ray source with a column density of $10^{23} \mathrm{~cm}^{-2}$ while the remaining part and the blackbody emission are attenuated by a reduced column density of $1.5 \times 10^{21} \mathrm{~cm}^{-2}$. Intensity variations of individual spectral components between spin minimum and maximum suggest that the spin modulation is mainly caused by viewing effects and only little changes in absorption. The X-ray spectrum of 1RXS J154814.5-452845 and in particular the detection of soft blackbody emission make this IP an interesting case between soft IPs and classical hard IPs. 
Acknowledgements. The ROSAT and XMM-Newton projects are supported by the German Bundesministerium für Bildung und Forschung / Deutsches Zentrum für Luft- und Raumfahrt (BMBF/DLR), the Max-Planck-Gesellschaft and the Heidenhain-Stiftung.

\section{References}

Aschenbach, B., Briel, U. G., Haberl, F., et al. 2000, in X-Ray Optics, Instruments, and Missions III, ed. J. E. Trümper, \& B. Aschenbach, SPIE, 4012, 731

Baraffe, I., \& Kolb, U. 2000, MNRAS, 318, 354

Beardmore, A. P., Osborne, J. P., \& Hellier, C. 2000, MNRAS, 315,307

Bertin, E., \& Arnouts, S. 1996, A\&AS, 117, 393

Beuermann, K., Baraffe, I., Kolb, U., \& Weichhold, M. 1998, A\&A, 339, 518

Beuermann, K., \& Burwitz, V. 1995, in ASP Conf. Ser., 85, Magnetic Cataclysmic Variables, 99

Beuermann, K., \& Thomas, H. 1993, Adv. Space Res., 13, 115

Bonnet-Bidaud, J. M., Mouchet, M., de Martino, D., Matt, G., \& Motch, C. 2001, A\&A, 374, 1003

Buccheri, R., Bennett, K., Bignami, G. F., et al. 1983, A\&A, 128,245

Buckley, D. A. H., Haberl, F., Motch, C., et al. 1997, MNRAS, 287,117

Burwitz, V., Haberl, F., Predehl, P., Reinsch, K., \& Barwig, H. 2001, in Yokohama Conf.

David, L., Harnden, J. F., Kearns, K., \& Zombeck, M. 1996, The ROSAT High Resolution Imager (HRI), USRSDC/SAO Calibration Rep., revised

de Martino, D., Matt, G., Mukai, K., et al. 2001, A\&A, 377, 499

Done, C., \& Magdziarz, P. 1998, MNRAS, 298, 737

Duck, S. R., Rosen, S. R., Ponman, T. J., et al. 1994, MNRAS, 271,372

Frank, J., King, A., \& Raine, D. 1992, Accretion Power in Astrophysics (Accretion Power in Astrophysics, ISBN 0521408636, Cambridge University Press, 1992)

Haberl, F., \& Motch, C. 1995, A\&A, 297, L37

Haberl, F., Thorstensen, J. R., Motch, C., et al. 1994, A\&A, 291,171
Hellier, C. 1995, in ASP Conf. Ser. 85, Magnetic Cataclysmic Variables, 185

Hellier, C. 1999, in ASP Conf. Ser. 157, Annapolis Workshop on Magnetic Cataclysmic Variables, 1

Hellier, C., Mason, K. O., Rosen, S. R., \& Cordova, F. A. 1987, MNRAS, 228, 463

Inoue, H. 1985, Space Sci. Rev., 40, 317

Jacoby, G. H., Hunter, D. A., \& Christian, C. A. 1984, ApJS, 56,257

Jansen, F., Lumb, D., Altieri, B., et al. 2001, A\&A, 365, L1

King, A. R., \& Lasota, J. P. 1990, MNRAS, 247, 214

Lomb, N. R. 1976, Ap\&SS, 39, 447

Mason, K. O., Watson, M. G., Ponman, T. J., et al. 1992, MNRAS, 258, 749

Matt, G., de Martino, D., Gänsicke, B. T., et al. 2000, A\&A, 358,177

Mauche, C. W. 2001, in The Physics of Cataclysmic Variables and related Objects, Göttingen August 2001

Mewe, R., Gronenschild, E. H. B. M., \& van den Oord, G. H. J. 1985, A\&AS, 62, 197

Motch, C., Belloni, T., Buckley, D., et al. 1991, A\&A, 246, L24

Motch, C., Haberl, F., Guillout, P., et al. 1996, A\&A, 307, 459

Panei, J. A., Althaus, L. G., \& Benvenuto, O. G. 2000, A\&A, 353,970

Patterson, J. 1994, PASP, 106, 209

Pfeffermann, E., Briel, U. G., Hippmann, H., et al. 1987, Proc. SPIE, 733, 519

Potter, S. B., Cropper, M., Mason, K. O., Hough, J. H., \& Bailey, J. A. 1997, MNRAS, 285, 82

ROSAT Consortium. 2000, ROSAT News, 72, 1

Scargle, J. D. 1982, ApJ, 263, 835

Smith, D. A., \& Dhillon, V. S. 1998, MNRAS, 301, 767

Strüder, L., Briel, U., Dennerl, K., et al. 2001, A\&A, 365, L18

Torres-Dodgen, A. V., \& Weaver, W. B. 1993, PASP, 105, 693

Trümper, J. 1982, Adv. Space Res., 2, 241

Turner, M. J. L., Abbey, A., Arnaud, M., et al. 2001, A\&A, 365, L27

Voges, W., Aschenbach, B., Boller, T., et al. 1999, A\&A, 349, 389

Warner, B. 1995, Cataclysmic variable stars (Cambridge Astrophysics Series, Cambridge, New York: Cambridge University Press)

Welsh, W. F., \& Martell, P. J. 1996, MNRAS, 282, 739 